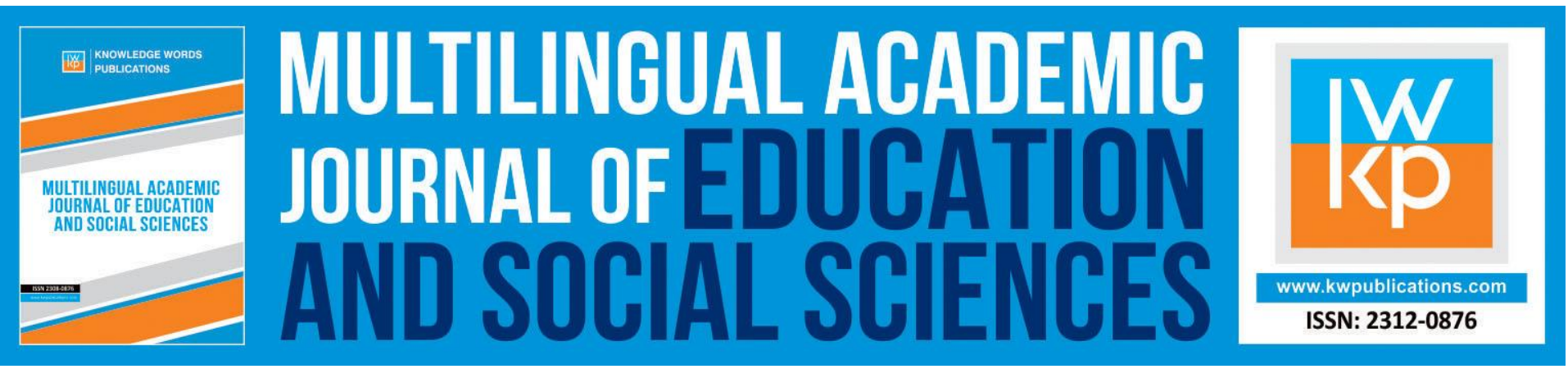

\title{
Multilingualism in the Cypriot Educational System: A Record of Primary School Students' Views and Attitudes
}

\author{
Panagiotis Panteli, Eleni Griva, Eleni Tsakiridou
}

To Link this Article: http://dx.doi.org/ 10.46886 /MAJESS/v1-i1/7286

DOI: 10.46886/MAJESS/v1-i1/7286

Received: 09 January 2013, Revised: 13 February 2013, Accepted: 08 March 2013

Published Online: 25 April 2013

In-Text Citation: (Panteli et al., 2013)

To Cite this Article: Panteli, P., Griva, E., \& Tsakiridou, E. (2013). Multilingualism in the Cypriot Educational System: A Record of Primary School Students' Views and Attitudes. Multilingual Academic Journal of Education and Social Sciences, 1(1), 19-38.

Copyright: (C) The Authors 2013

Published by Knowledge Words Publications (www.kwpublications.com)

This article is published under the Creative Commons Attribution (CC BY 4.0) license. Anyone may reproduce, distribute, translate and create derivative works of this article (for both commercial and non-commercial purposes), subject to full attribution to the original publication and authors. The full terms of this license may be seen at: http://creativecommons.org/licences/by/4.0/legalcode

Vol. 1, No. 1, 2013, Pg. 19 - 38

https://kwpublications.com/journals/journaldetail/MAJESS

JOURNAL HOMEPAGE

Full Terms \& Conditions of access and use can be found at https://kwpublications.com/pages/detail/publication-ethics 


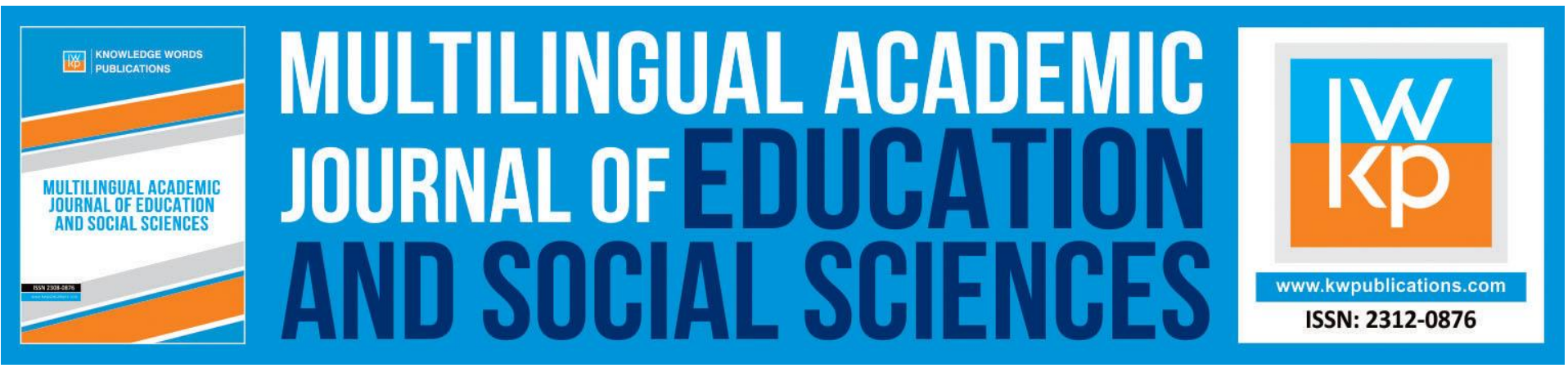

\title{
Multilingualism in the Cypriot Educational System: A Record of Primary School Students' Views and Attitudes
}

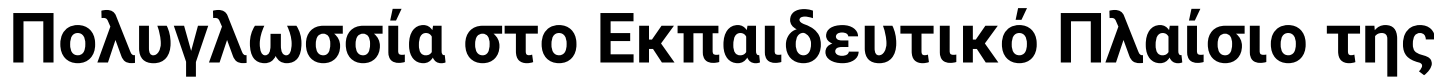

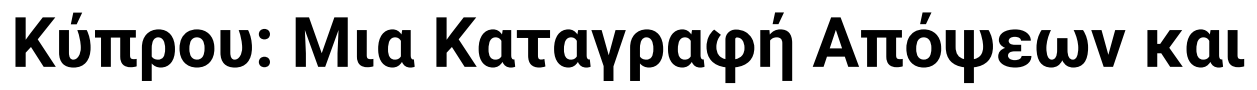

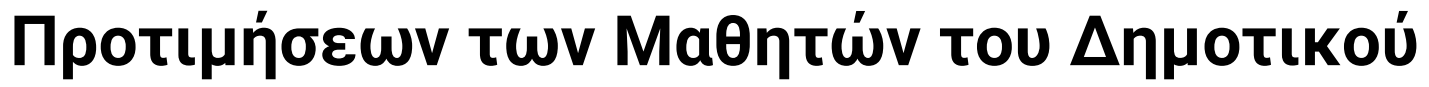

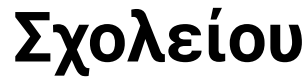

\author{
Panagiotis Panteli, Eleni Griva, Eleni Tsakiridou \\ Assistant Professor at the Faculty of Education - University of Western Macedonia, \\ Department of Primary Education of the University of Western Macedonia in Greece.
}

\begin{abstract}
The present study was conducted with the aim to provide an account of primary school students' attitudes and views on issues related to English language learning and multilingual learning in the Cypriot Educational System. The stimulus for conducting the study can be identified in current EU multilingual policy, the spread of English world-wide, and the changing status of English within Europe, along with the scarcity of research concerning students' views and preferences in the Cypriot context. In particular, the study aimed at:

a. identifying primary school students' attitudes towards English and other European Languages

b. identifying their perceptions of early foreign language learning

c. identifying their attitudes towards Turkish language

d. specifying their perceptions of issues on multilingual education.

In total 1265 Cypriot students of 5th and 6th primary school grades participated in the study, which was conducted in the academic year 2010-2011. The research project utilised a questionnaire as a basic instrument to collect information from primary students. The findings indicated that the majority of the students acknowledged the dominant role of the English language and showed a positive attitude towards early language learning. Although they highlighted their enthusiasm for acquiring multilingual competence in 'strong' European languages, they expressed their unwillingness to learn Turkish language. Concluding, it is
\end{abstract}


MULTILINGUAL ACADEMIC JOURNAL OF EDUCATION AND SOCIAL SCIENCES

Vol. 1 No. 1, 2013, E-ISSN: 2308-0876 @ 2013 KWP

suggested that, despite the dominant role of English language, every effort should be made towards adopting educational policies and practices which support and foster multilingualism. Keywords: Multilingualism, English Language, Primary School Students, Attitudes, Views.

\section{Introduction}

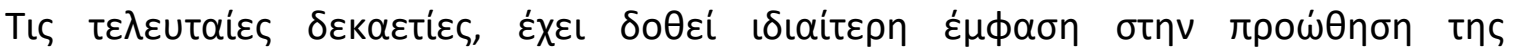

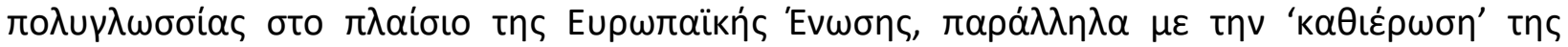

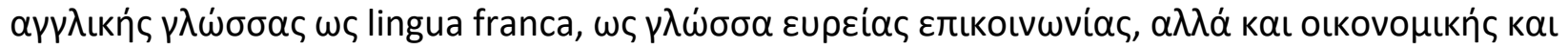

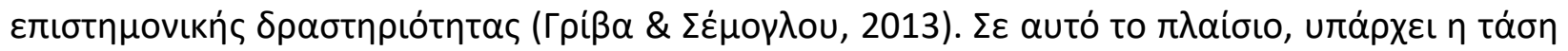

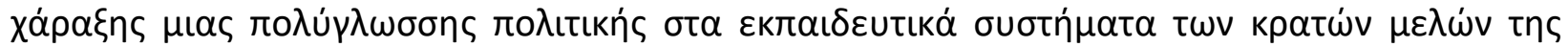

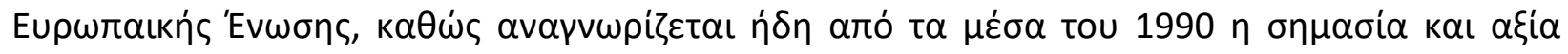

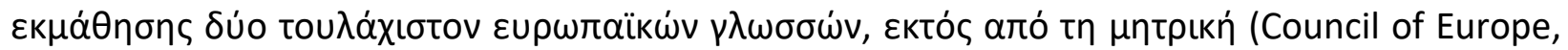

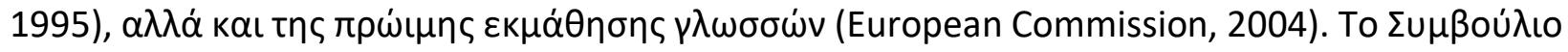

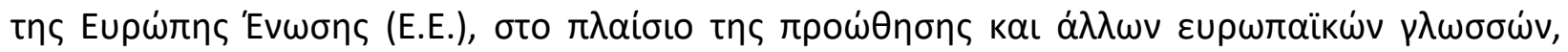

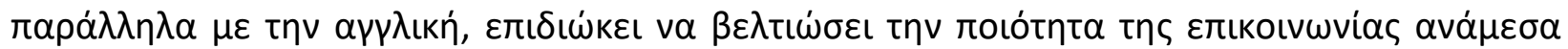

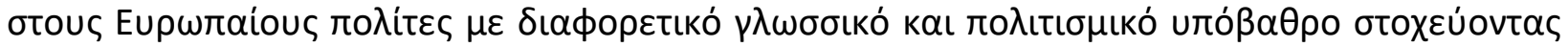

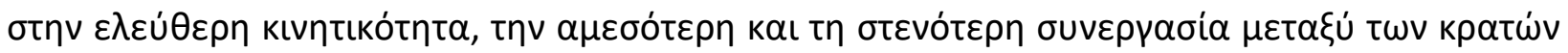
$\mu \varepsilon \lambda \omega \dot{v} v(\Gamma \rho i \beta \alpha, 2011: 58)$.

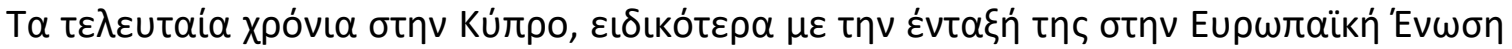

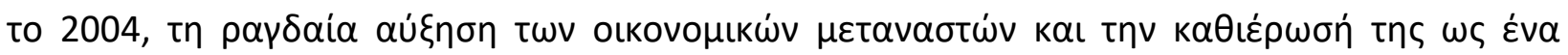

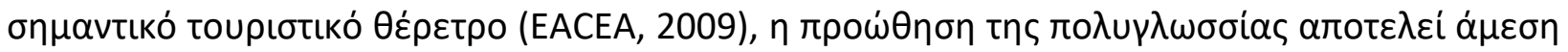

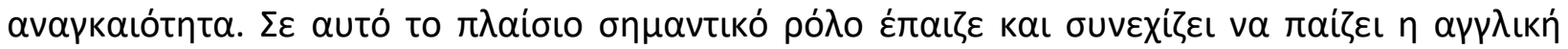

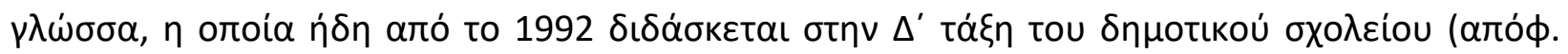

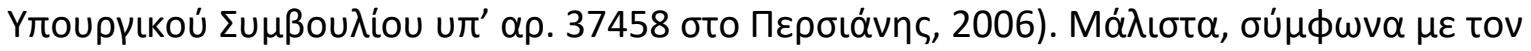

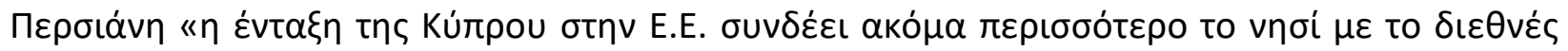

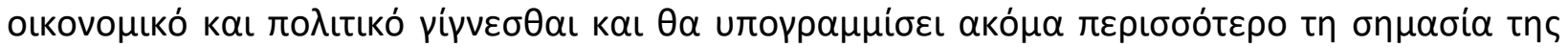

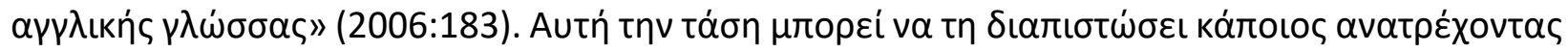

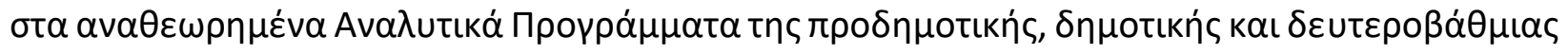

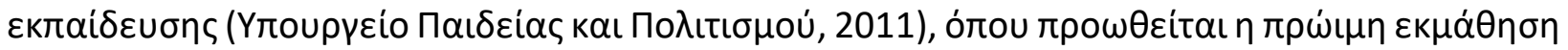

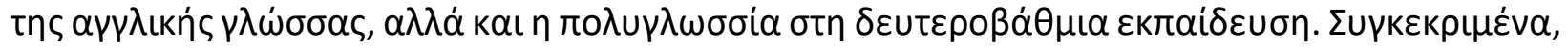

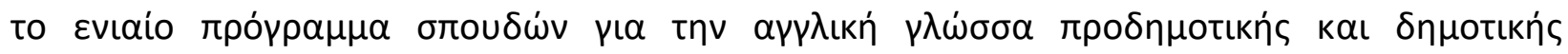

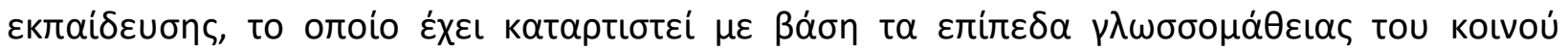

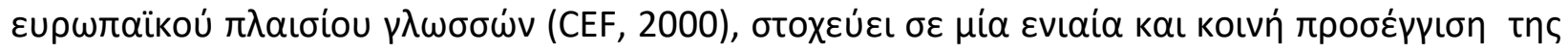

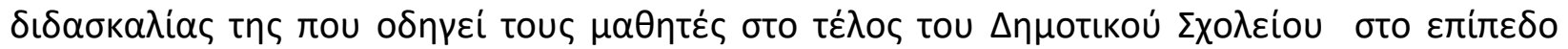

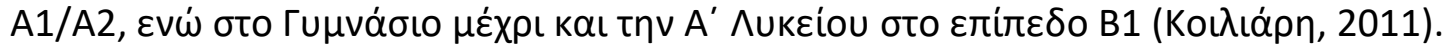

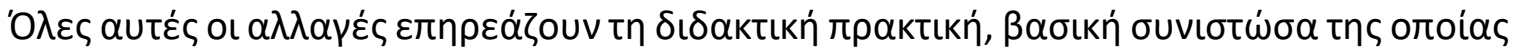

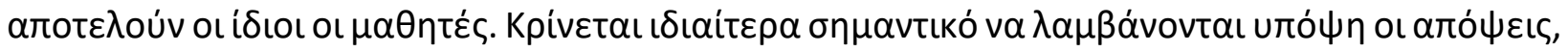

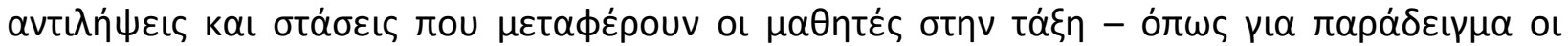

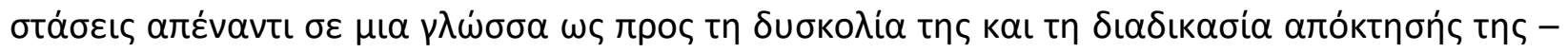

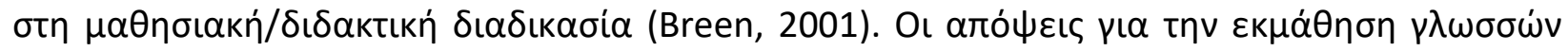

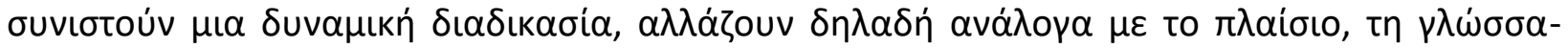
бтóxo к $\lambda \pi$ (Sakui \& Gaies, 1999). 
MULTILINGUAL ACADEMIC JOURNAL OF EDUCATION AND SOCIAL SCIENCES

Vol. 1 No. 1, 2013, E-ISSN: 2308-0876 @ 2013 KWP

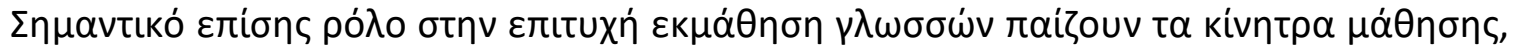

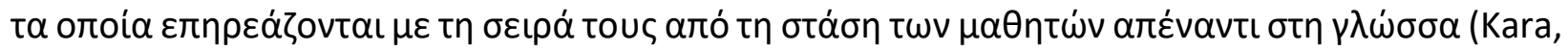

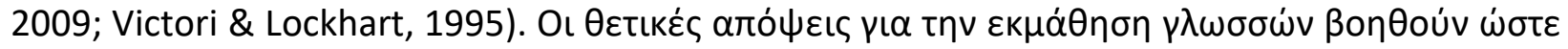

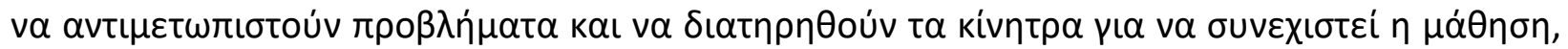

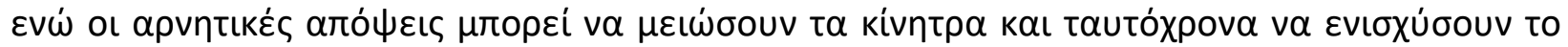

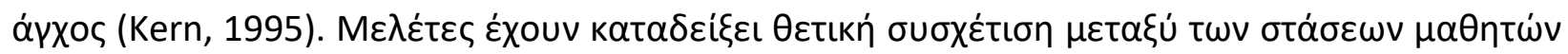

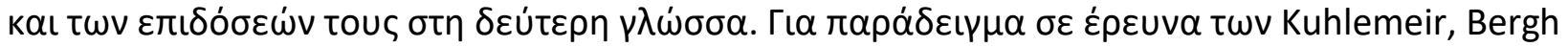

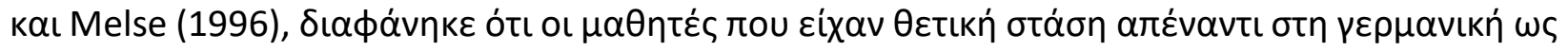

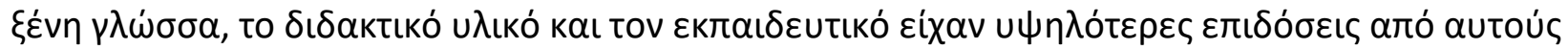

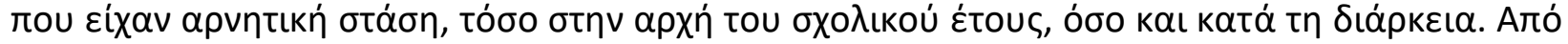

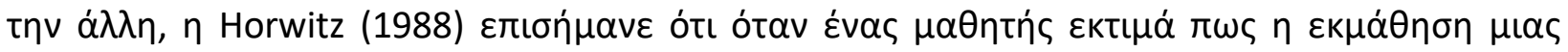

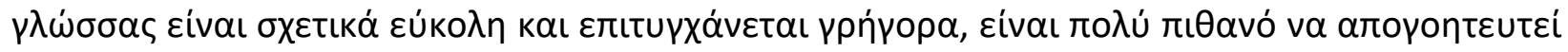

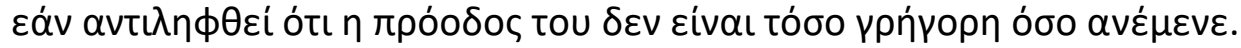

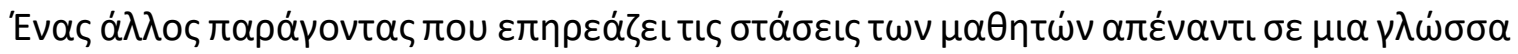

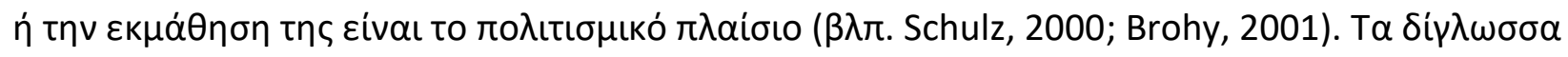

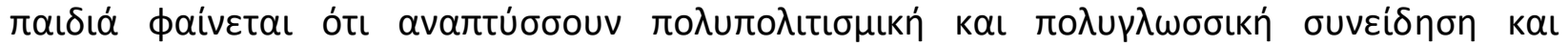
$\varepsilon \cup \alpha \iota \sigma \theta \eta \sigma i \alpha, \mu \varepsilon$ a (Edelenbos, Johnstone \& Kubanek, 2006; Griva \& Chostelidou, 2011).

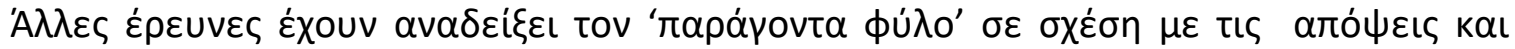

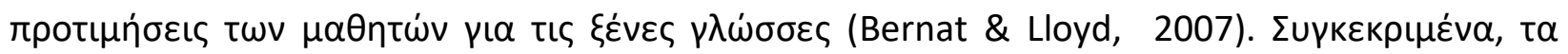

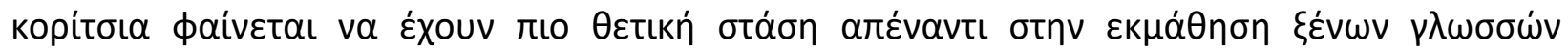

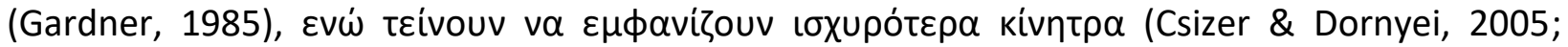

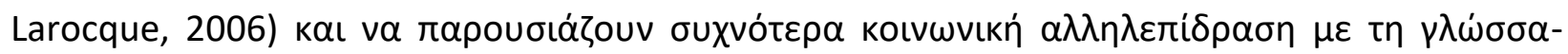

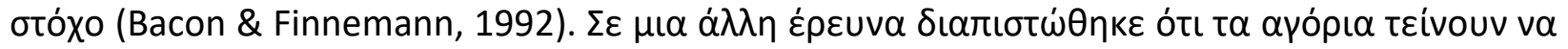

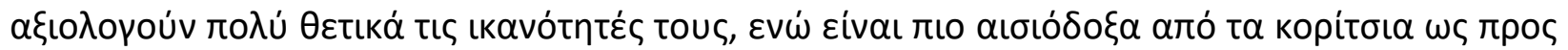

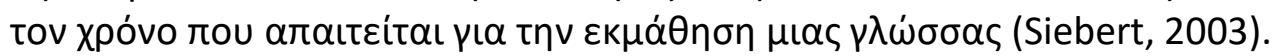

\section{H'Ep\&uva}

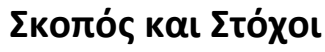

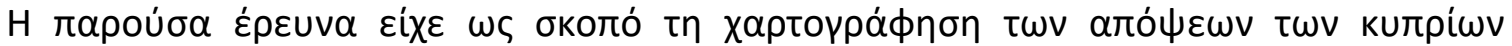

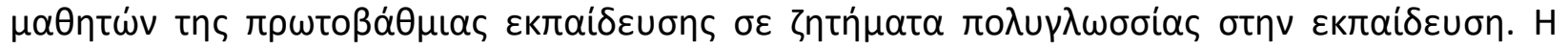

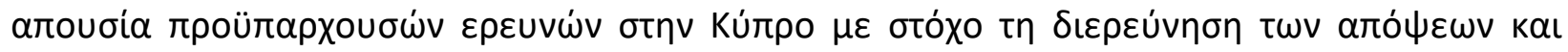

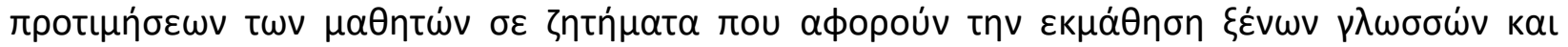

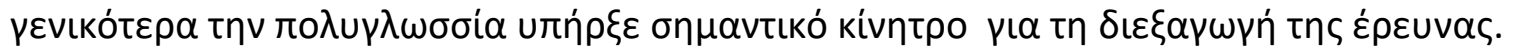

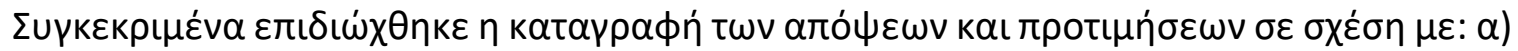

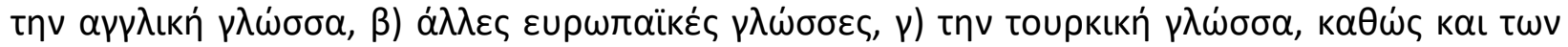

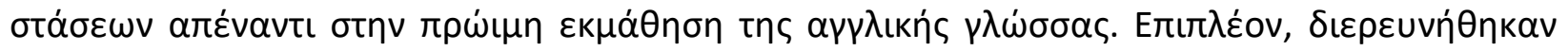

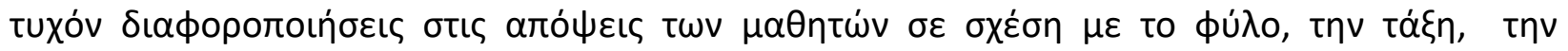

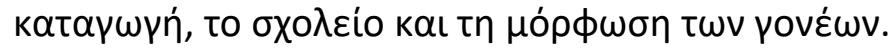

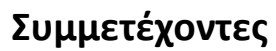

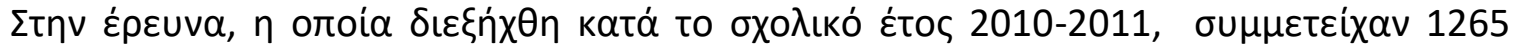

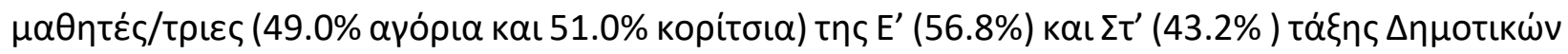

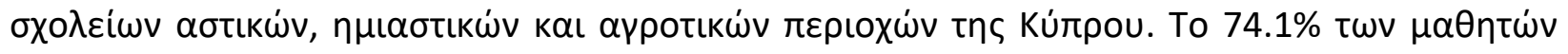




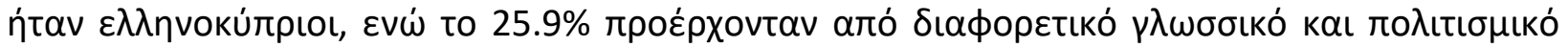

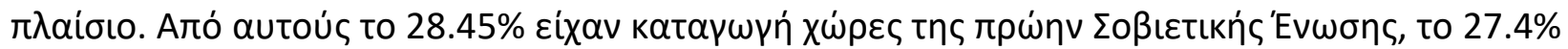

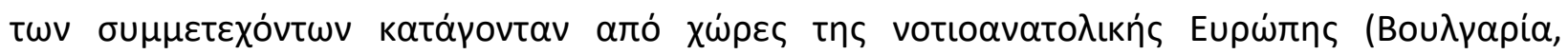

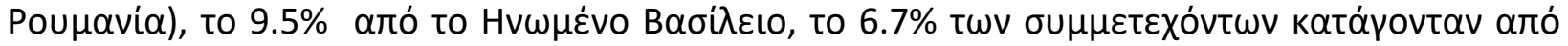

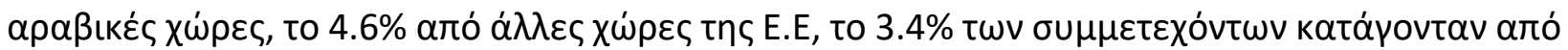

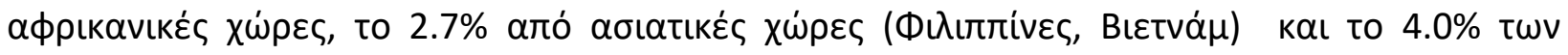

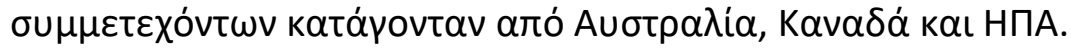

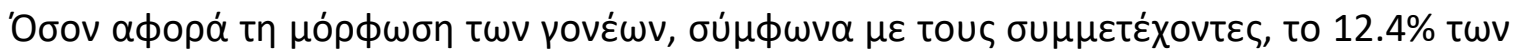

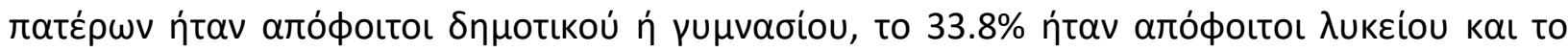

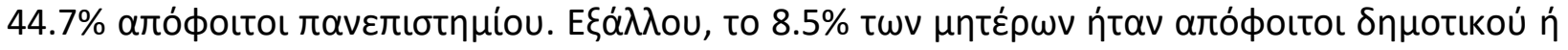

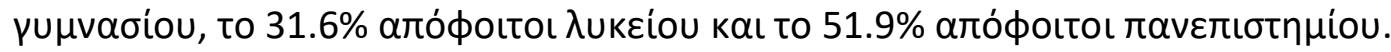

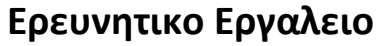

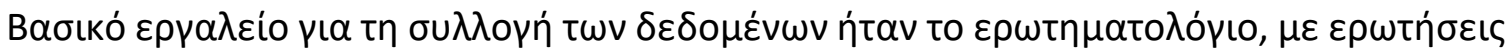

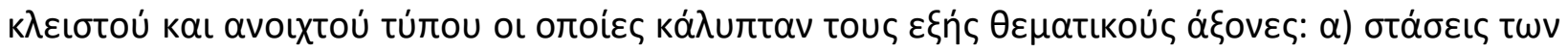

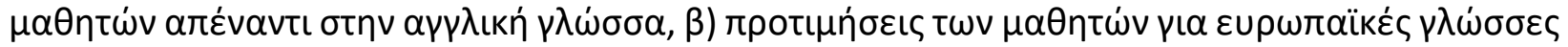

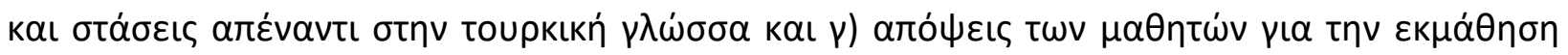

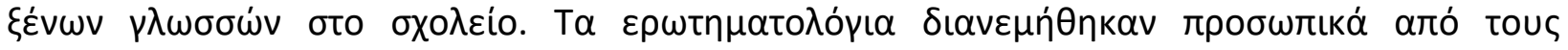

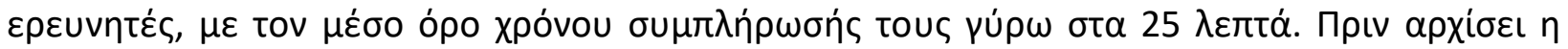

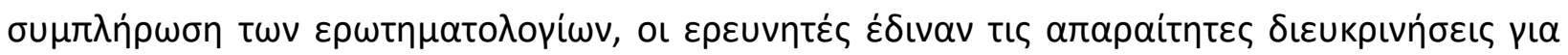

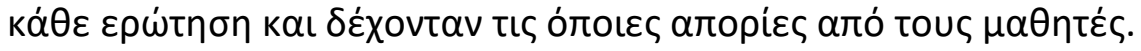

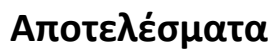

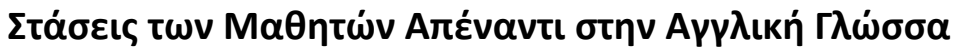

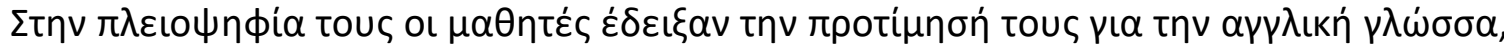

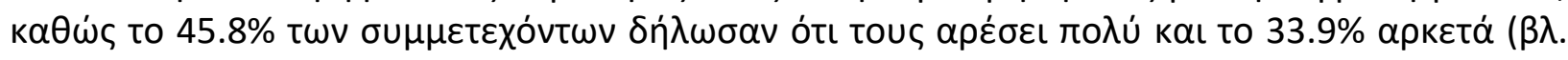

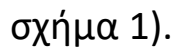


MULTILINGUAL ACADEMIC JOURNAL OF EDUCATION AND SOCIAL SCIENCES

Vol. 1 No. 1, 2013, E-ISSN: 2308-0876 @ 2013 KWP

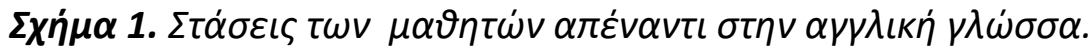

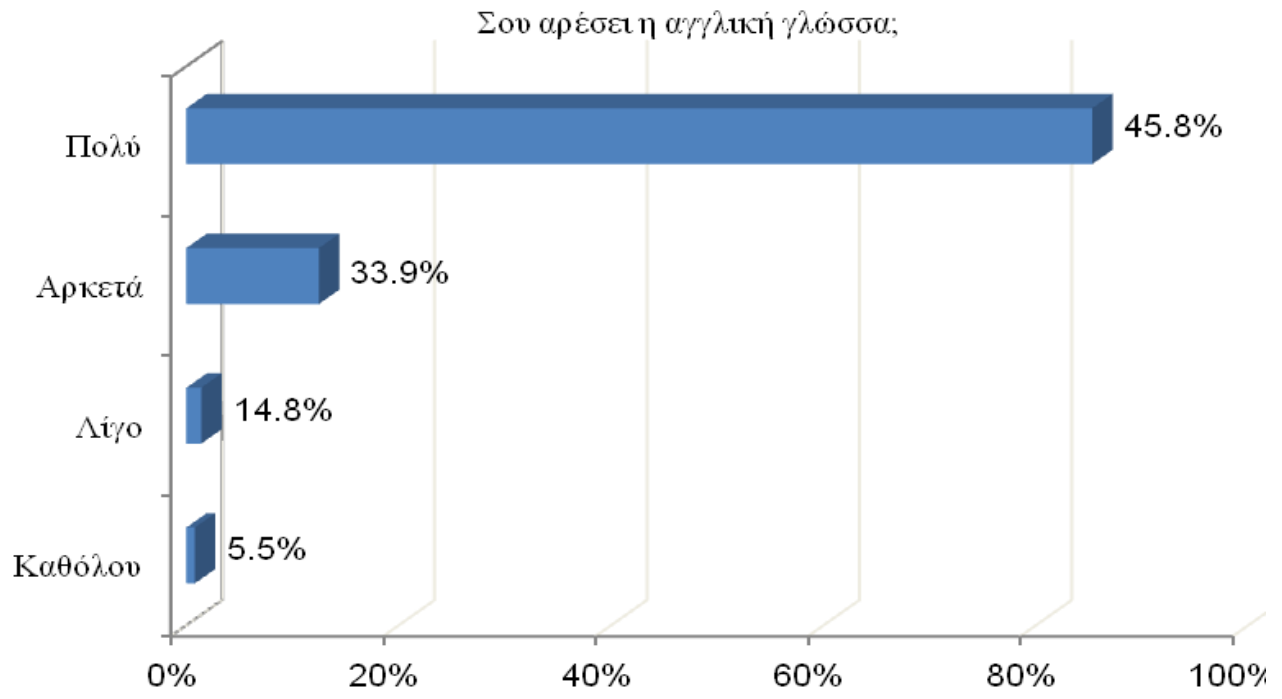

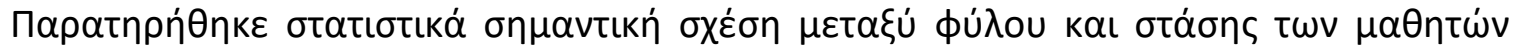

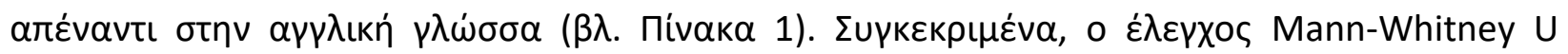

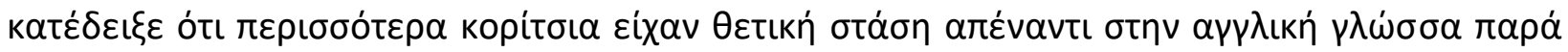

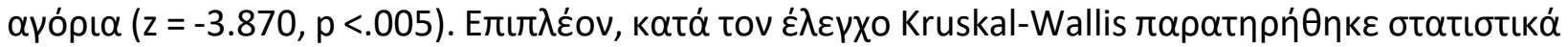

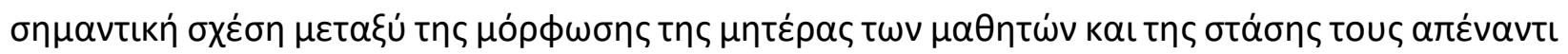

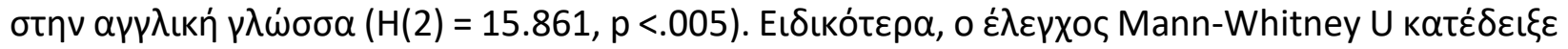

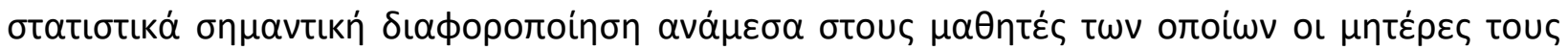

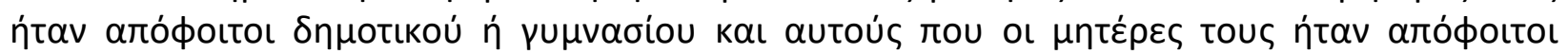

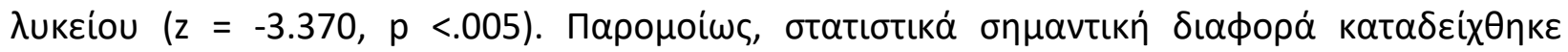

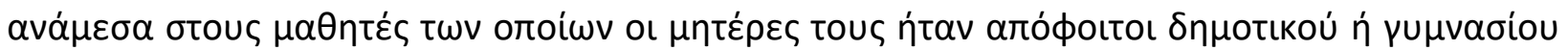

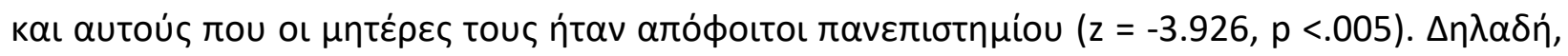

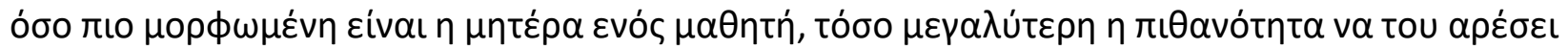

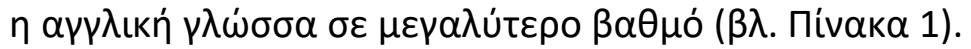


MULTILINGUAL ACADEMIC JOURNAL OF EDUCATION AND SOCIAL SCIENCES

Vol. 1 No. 1, 2013, E-ISSN: 2308-0876 @ 2013 KWP

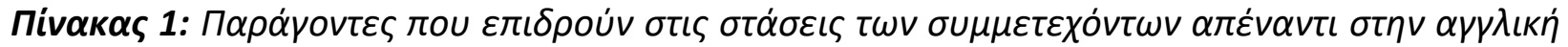
$\gamma \lambda \omega \dot{\omega} \sigma \sigma \alpha$

\begin{tabular}{|c|c|c|c|c|c|}
\hline \multirow{2}{*}{ 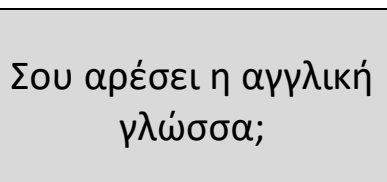 } & \multicolumn{2}{|c|}{ 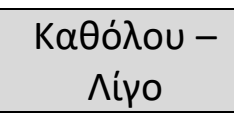 } & \multicolumn{2}{|c|}{ 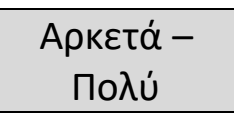 } & \multirow[b]{2}{*}{ 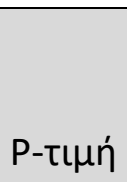 } \\
\hline & $\mathrm{N}$ & $\%$ & $\mathrm{~N}$ & $\%$ & \\
\hline \multicolumn{6}{|l|}{ Фú̀o } \\
\hline Aүópı $\alpha$ & 153 & 24.8 & 464 & 75.2 & \\
\hline Корі́тбı $\alpha$ & 103 & 16.0 & 540 & 84.0 & $<0.005$ \\
\hline \multicolumn{6}{|l|}{ Мó $\varphi \omega \sigma \eta \mu \eta \tau \varepsilon ́ \rho \alpha \varsigma$} \\
\hline 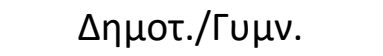 & 37 & 34.6 & 70 & 65.4 & \\
\hline 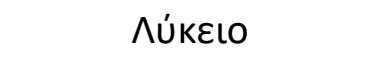 & 77 & 19.3 & 323 & 80.8 & \\
\hline 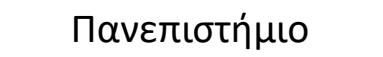 & 118 & 18.1 & 535 & 81.9 & $<0.005$ \\
\hline
\end{tabular}

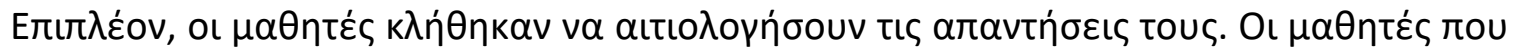

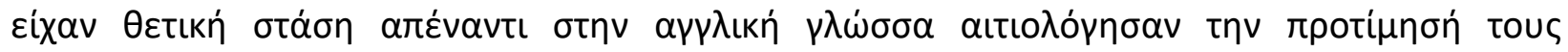

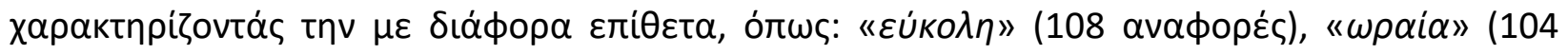

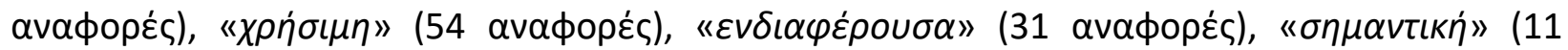

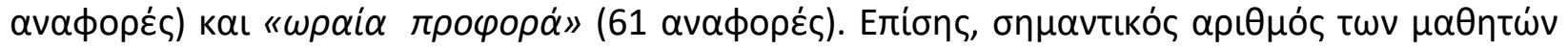

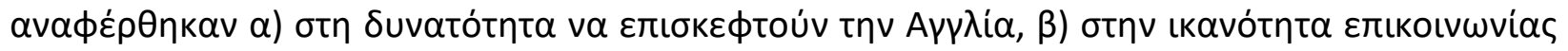

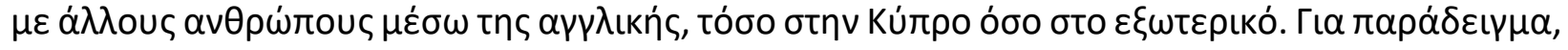

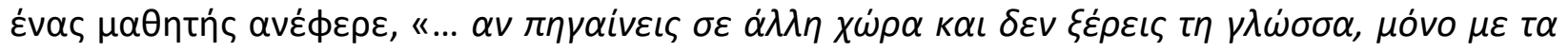

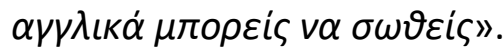

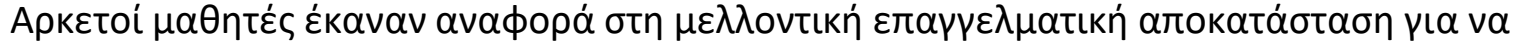

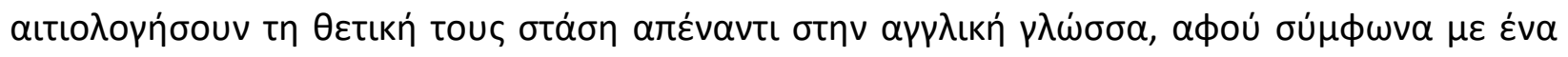

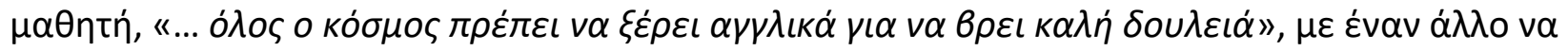

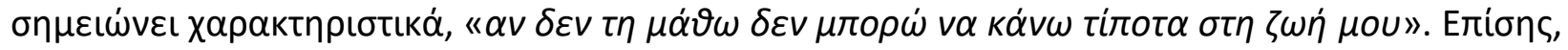

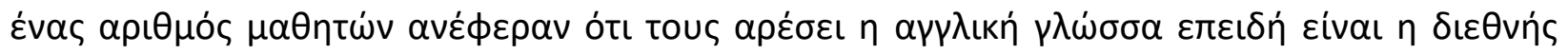

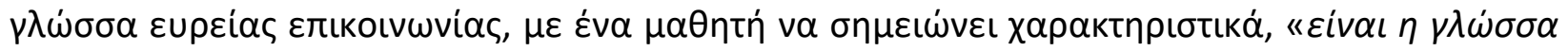

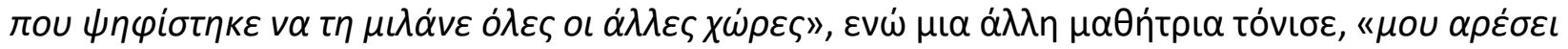

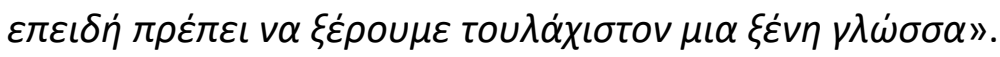

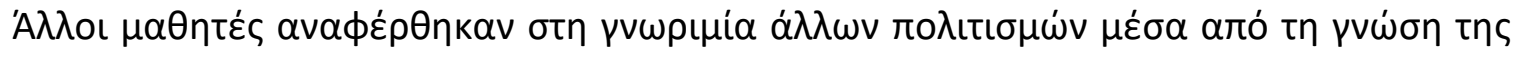

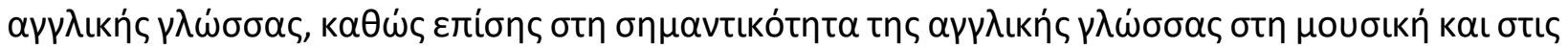

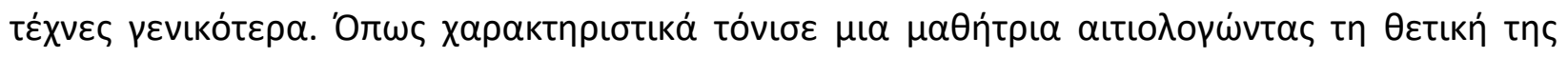

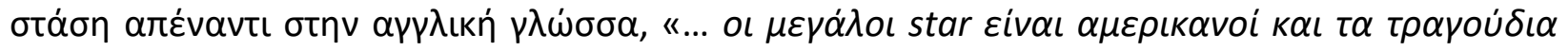

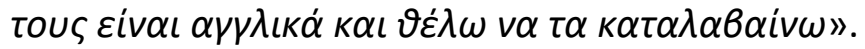

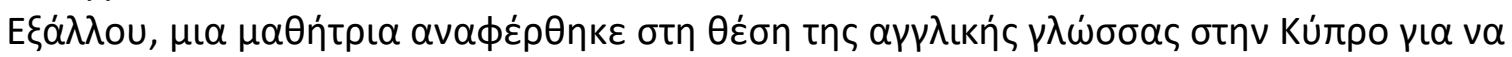

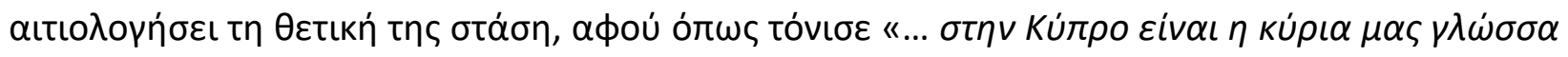

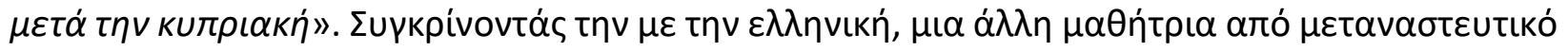


MULTILINGUAL ACADEMIC JOURNAL OF EDUCATION AND SOCIAL SCIENCES

Vol. 1 No. 1, 2013, E-ISSN: 2308-0876 @ 2013 KWP

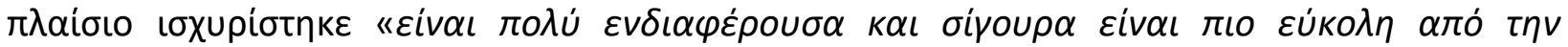

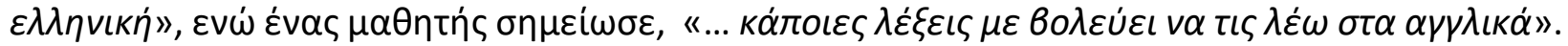

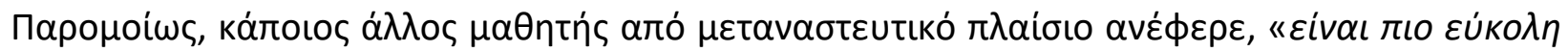

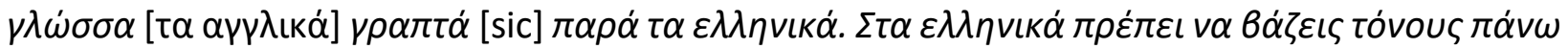

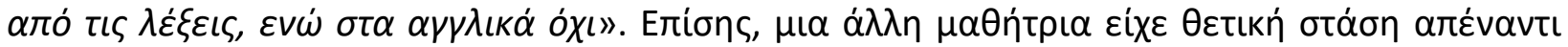

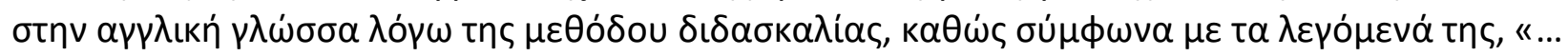

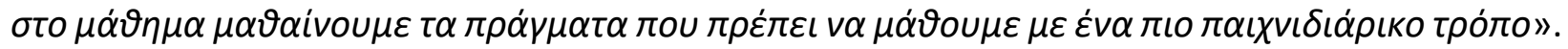

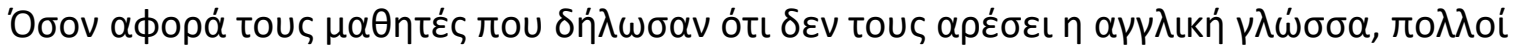

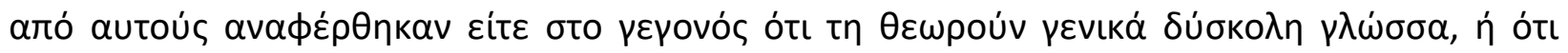

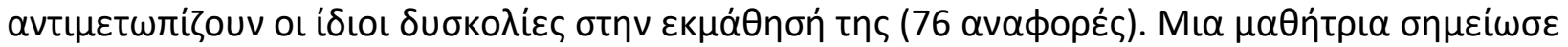

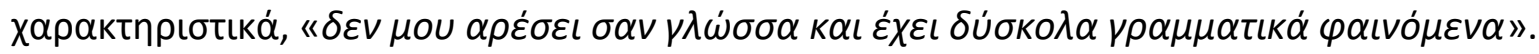

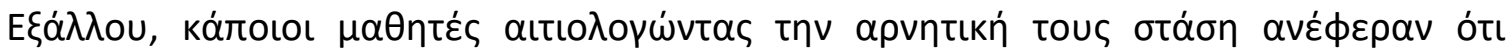

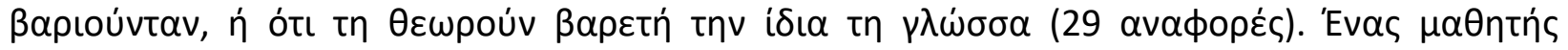

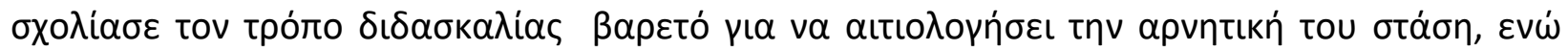

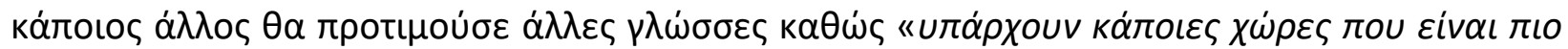

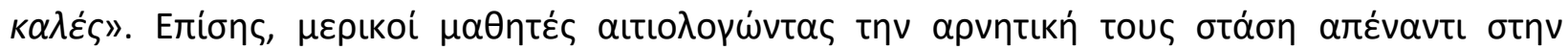

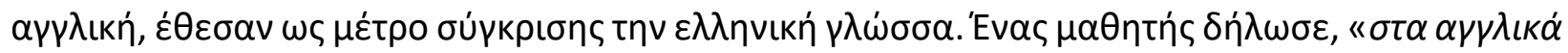

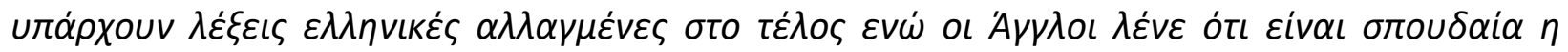

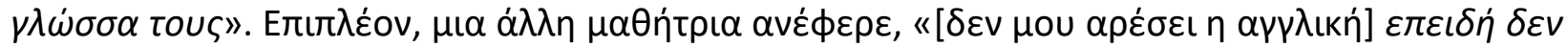

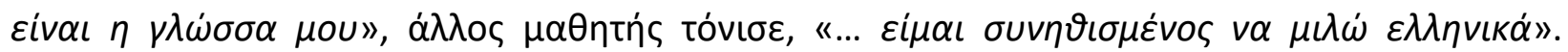

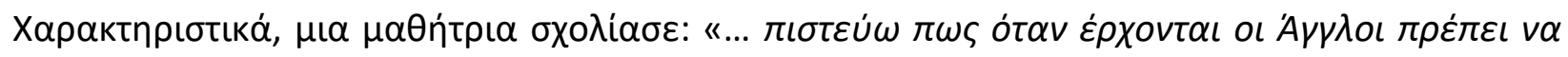

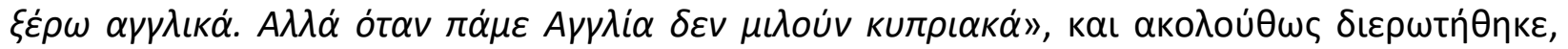

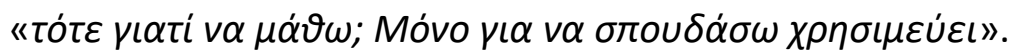

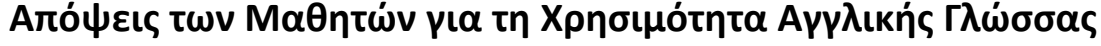

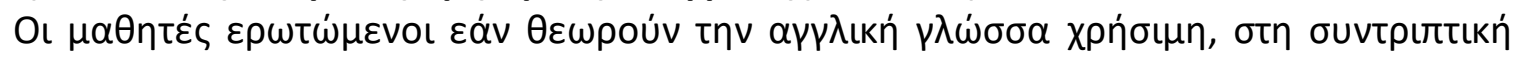

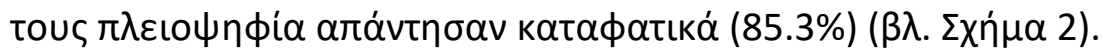

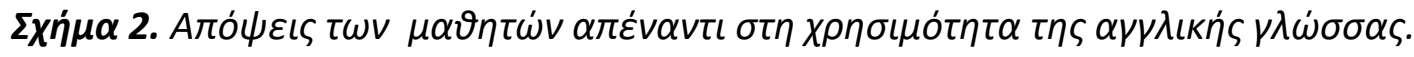

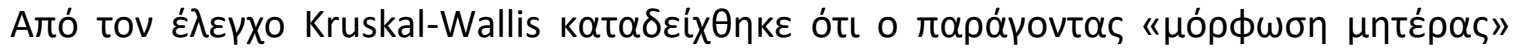

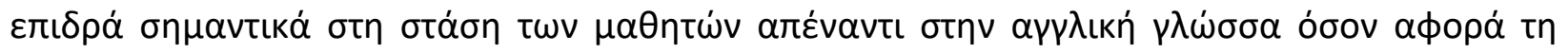

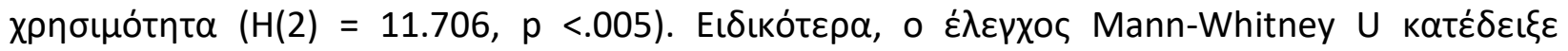

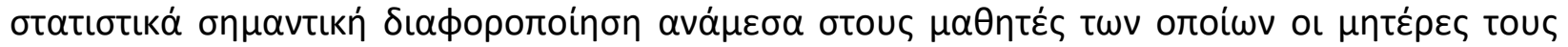

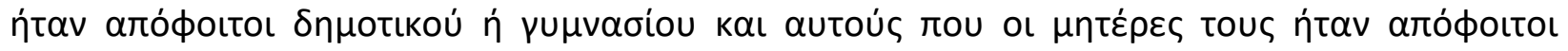

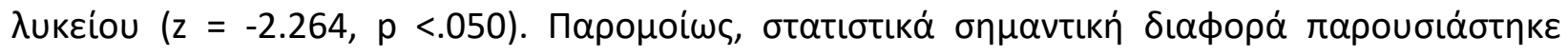

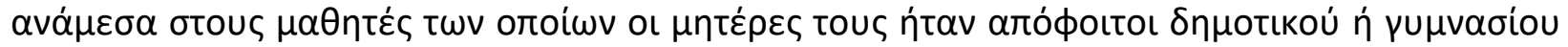

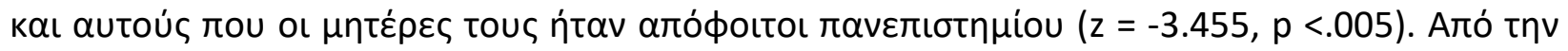

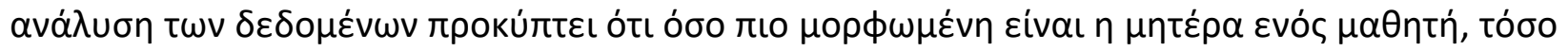

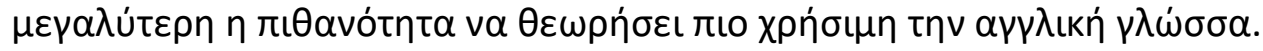

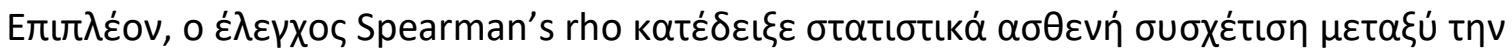

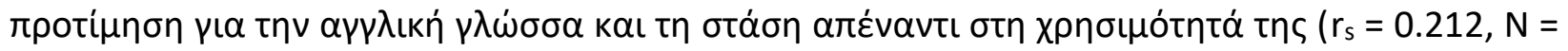

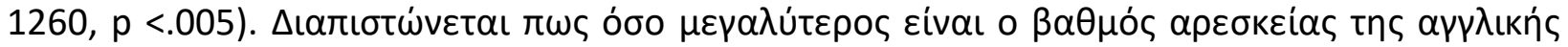

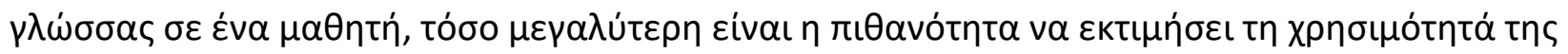

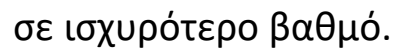


MULTILINGUAL ACADEMIC JOURNAL OF EDUCATION AND SOCIAL SCIENCES

Vol. 1 No. 1, 2013, E-ISSN: 2308-0876 @ 2013 KWP

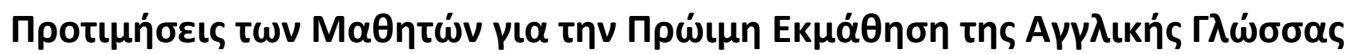

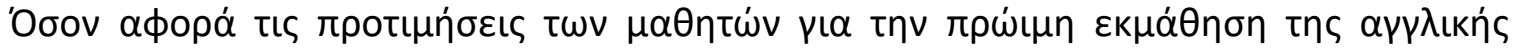

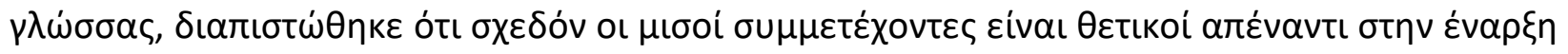

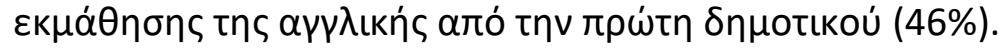

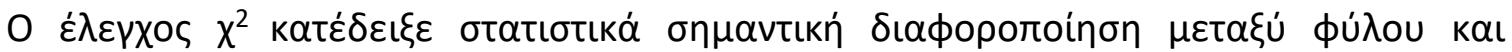

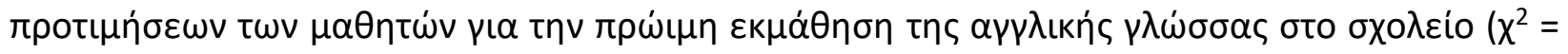

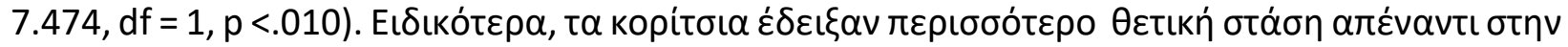

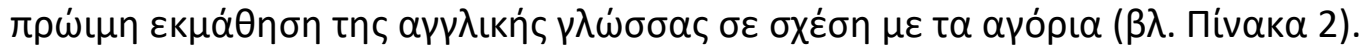

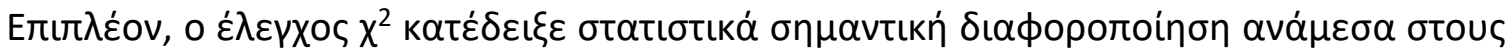

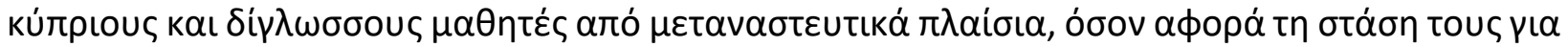

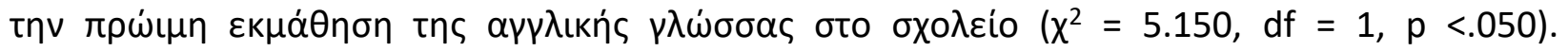

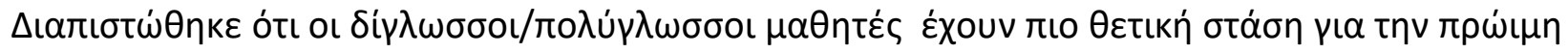

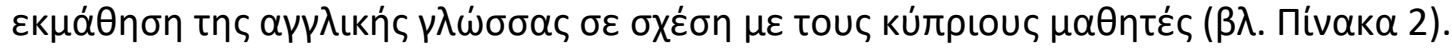

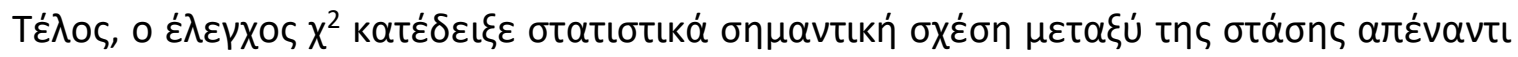

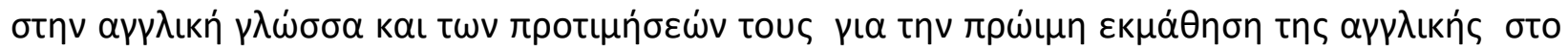

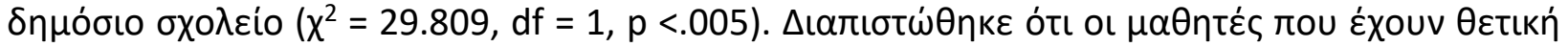

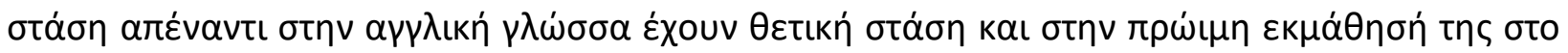
$\sigma \chi 0 \lambda \varepsilon$ ío.

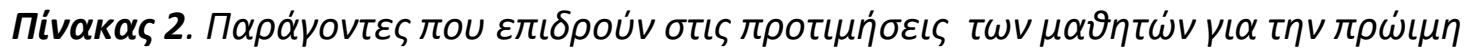

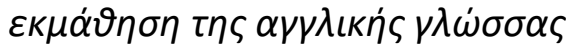

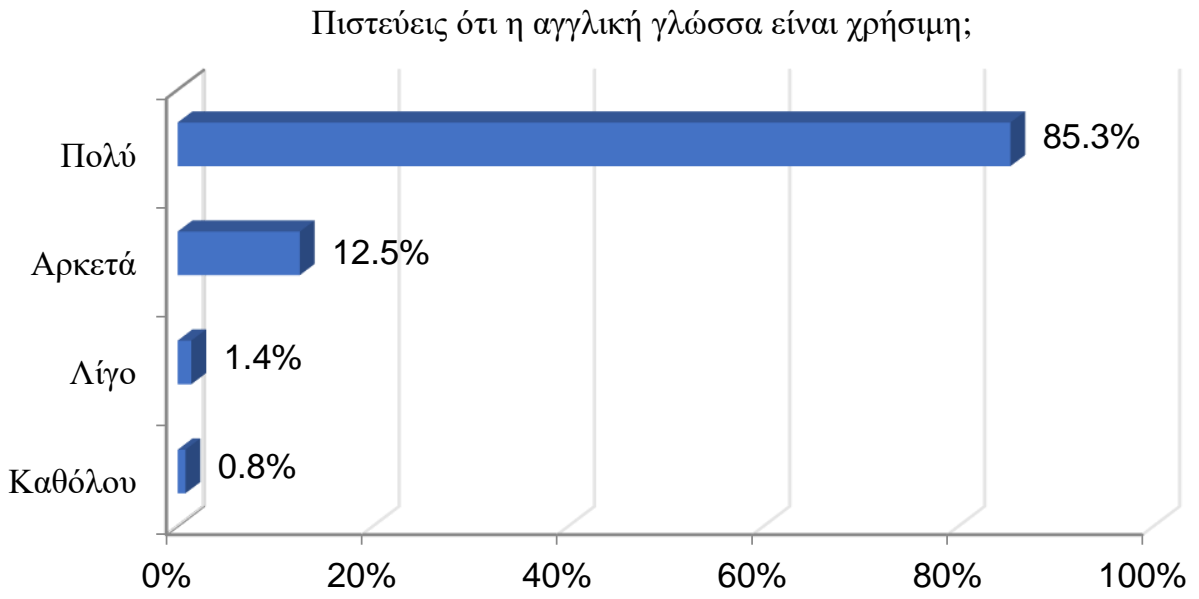


MULTILINGUAL ACADEMIC JOURNAL OF EDUCATION AND SOCIAL SCIENCES

Vol. 1 No. 1, 2013, E-ISSN: 2308-0876 @ 2013 KWP

\begin{tabular}{|c|c|c|c|c|c|}
\hline \multirow{2}{*}{ 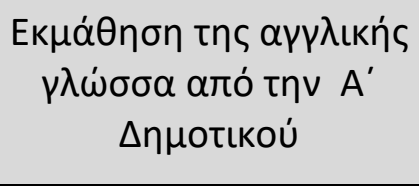 } & \multicolumn{2}{|c|}{$\mathrm{Nal}$} & \multicolumn{2}{|c|}{ OXı } & \multirow[b]{2}{*}{ 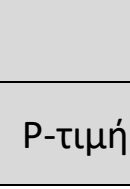 } \\
\hline & $\mathrm{N}$ & $\%$ & $\mathrm{~N}$ & $\%$ & \\
\hline \multicolumn{6}{|l|}{ Фú̀o } \\
\hline Aүópı & 256 & $\begin{array}{c}42 . \\
0\end{array}$ & $\begin{array}{c}35 \\
3\end{array}$ & 58.0 & \\
\hline Корі́тбıа & 316 & $\begin{array}{c}49 . \\
8 \\
\end{array}$ & $\begin{array}{c}31 \\
9 \\
\end{array}$ & 50.2 & $<.010$ \\
\hline \multicolumn{6}{|l|}{$\Gamma \lambda \omega \sigma \sigma \iota \alpha^{\prime} \pi \rho \circ \varphi і \lambda$} \\
\hline Kúrрıı & 429 & $\begin{array}{c}44 . \\
3\end{array}$ & $\begin{array}{c}54 \\
0\end{array}$ & 55.7 & \\
\hline $\begin{array}{c}\Delta \mathrm{i} \gamma \lambda \omega \sigma \sigma o \mathrm{l} \\
\pi \circ \lambda \dot{\gamma}\rangle \omega \omega \sigma \sigma \mathrm{l}\end{array}$ & 143 & $\begin{array}{c}52 . \\
0\end{array}$ & $\begin{array}{c}13 \\
2\end{array}$ & 48.0 & $<0.050$ \\
\hline
\end{tabular}

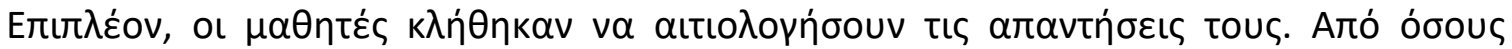

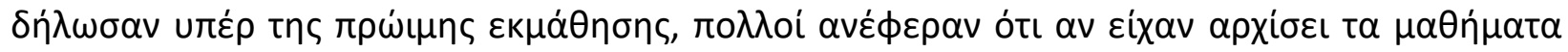

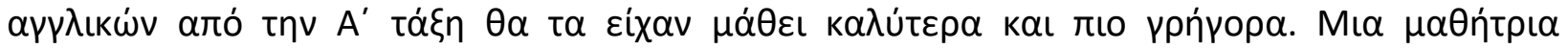

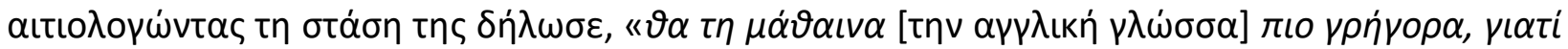

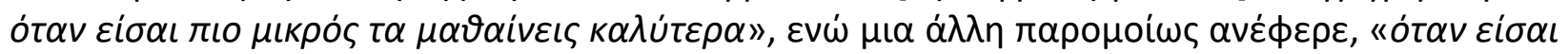

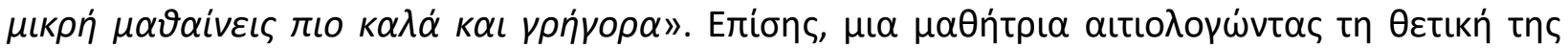

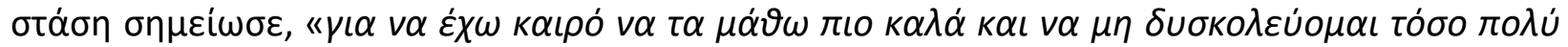
$\tau \omega \dot{\omega} \rho \alpha »$.

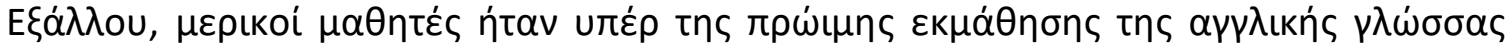

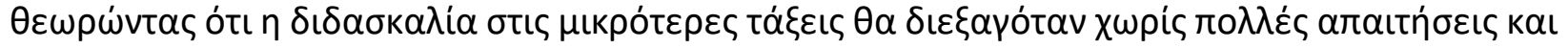

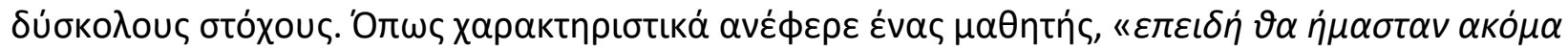

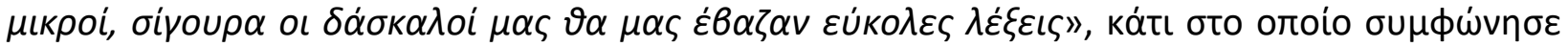

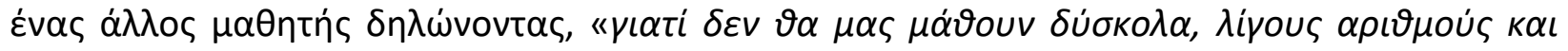

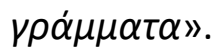

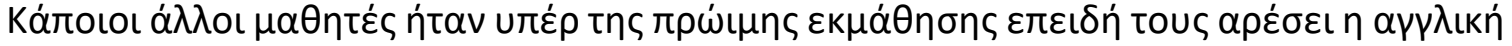

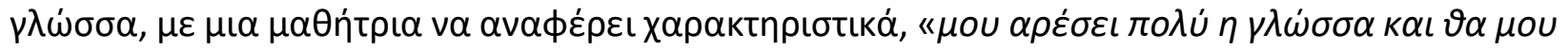

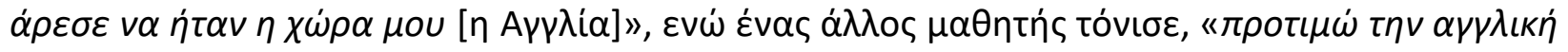

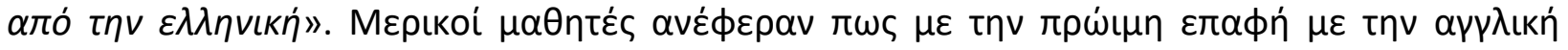

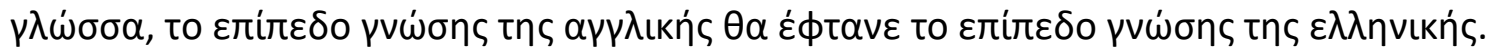


MULTILINGUAL ACADEMIC JOURNAL OF EDUCATION AND SOCIAL SCIENCES

Vol. 1 No. 1, 2013, E-ISSN: 2308-0876 @ 2013 KWP

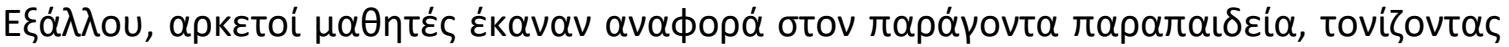

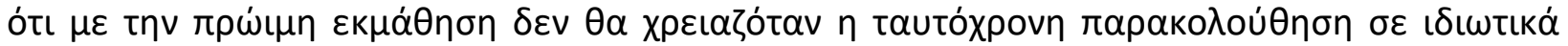

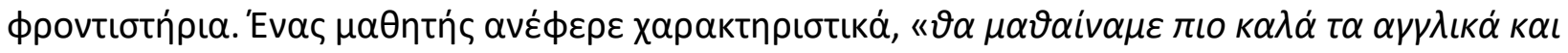

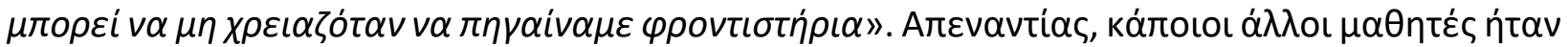

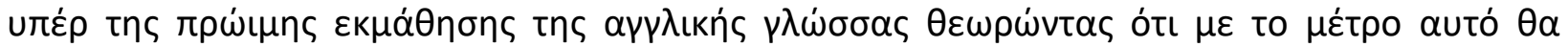

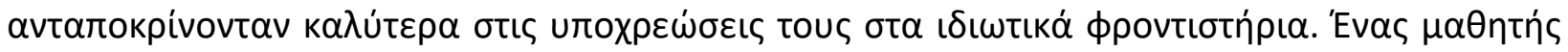

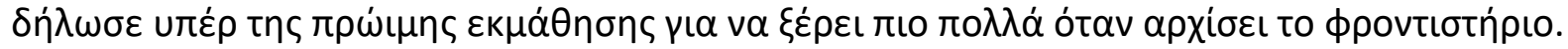

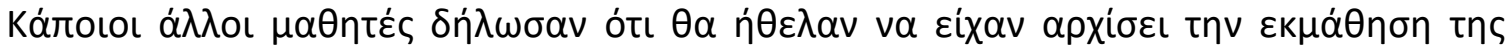

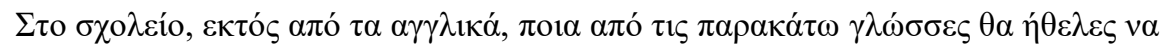
$\mu \alpha \dot{\theta} \theta \varepsilon 1 \zeta$;

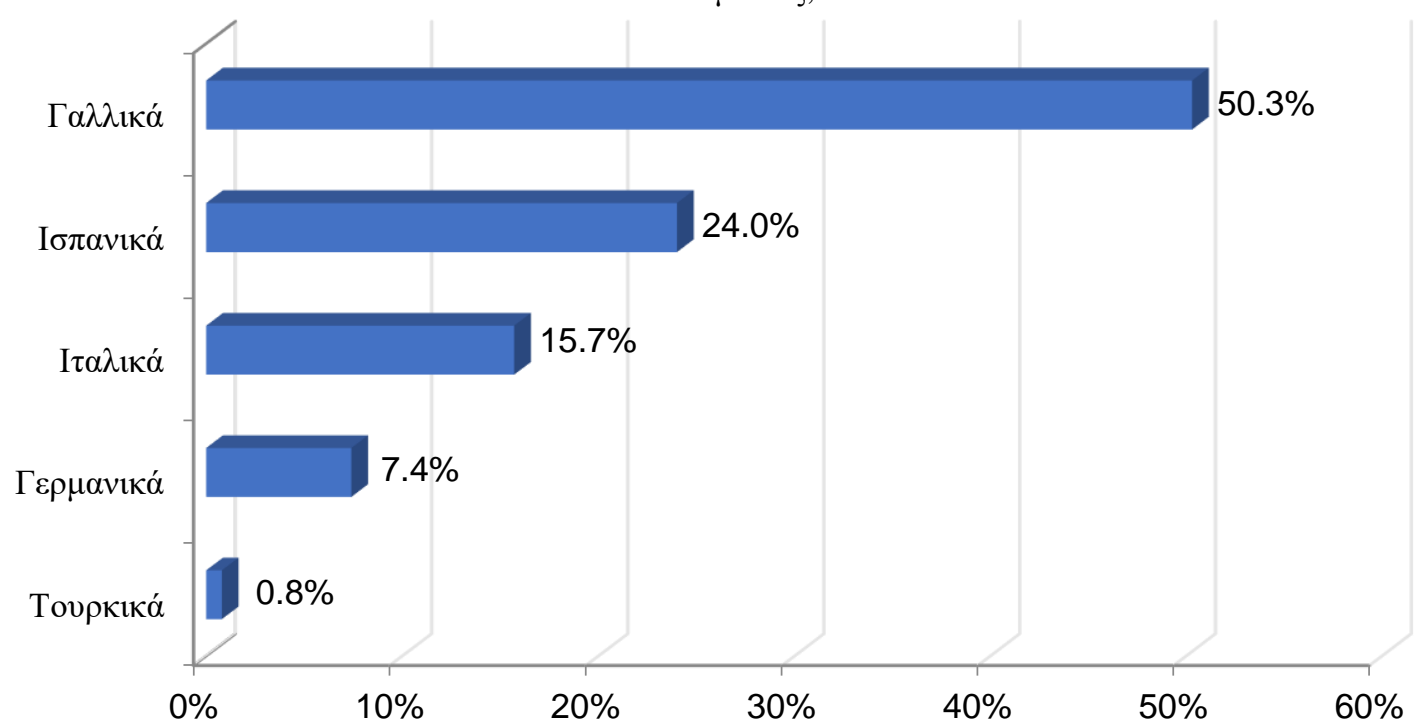

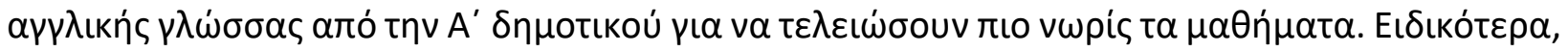

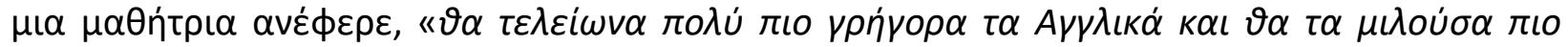

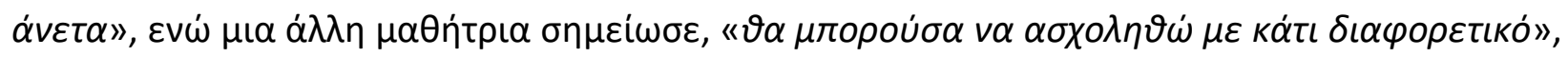

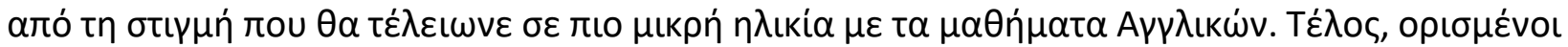

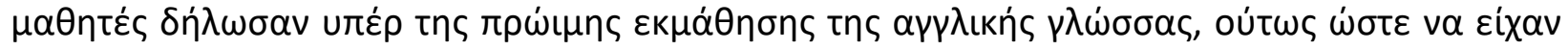

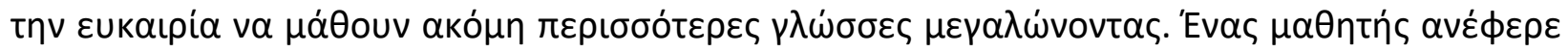

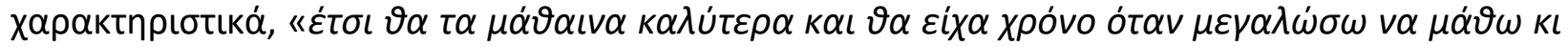
$\dot{\alpha} \lambda \lambda \varepsilon \varsigma \gamma \lambda \hat{\omega} \sigma \sigma \varepsilon \varsigma »$.

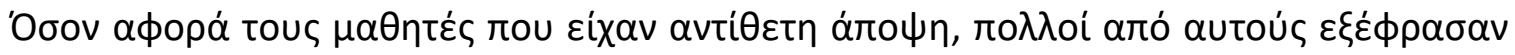

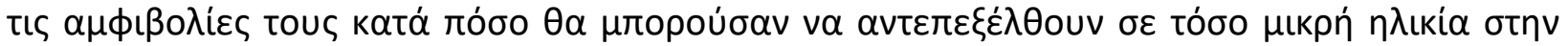

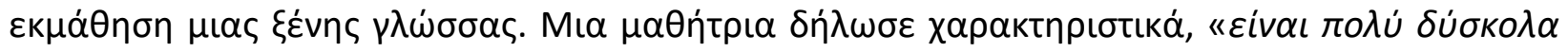

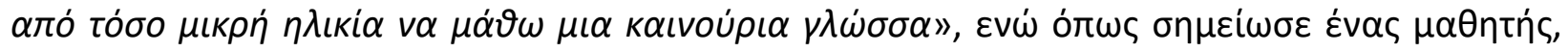

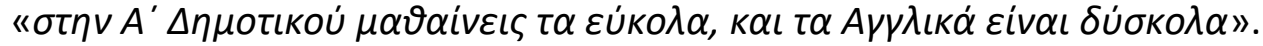

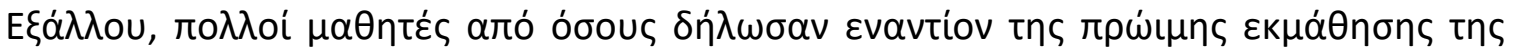

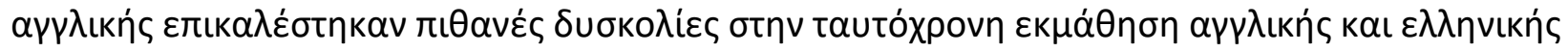

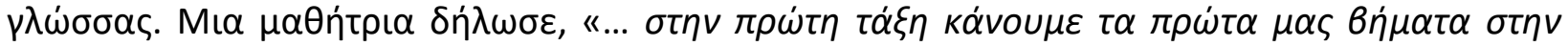

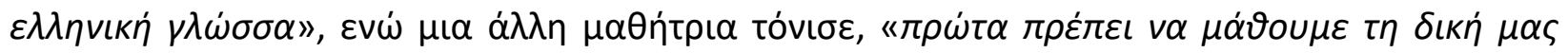




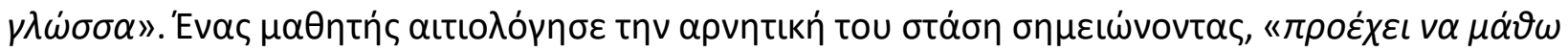

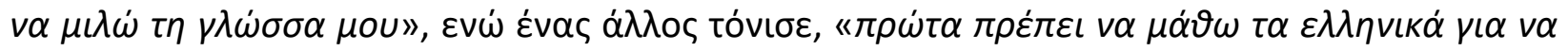

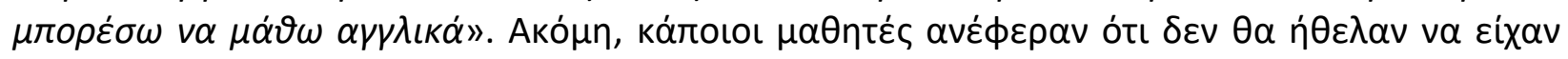

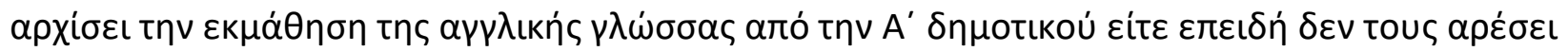

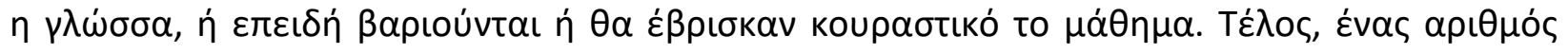

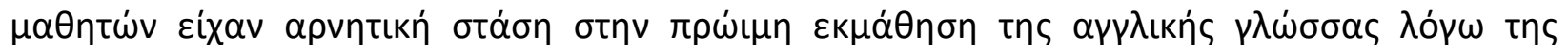

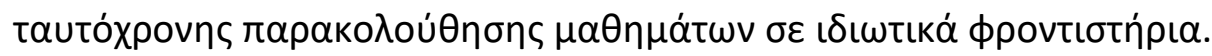

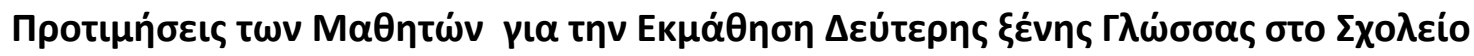

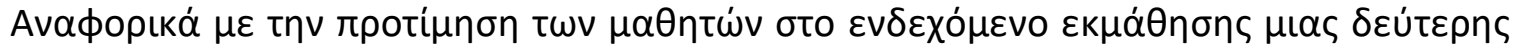

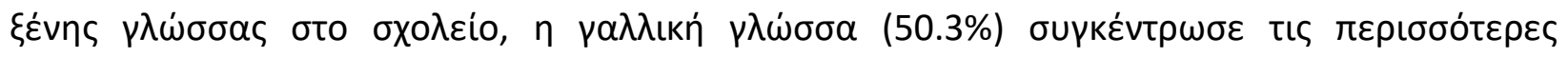

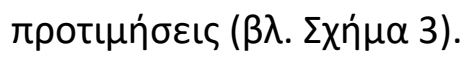

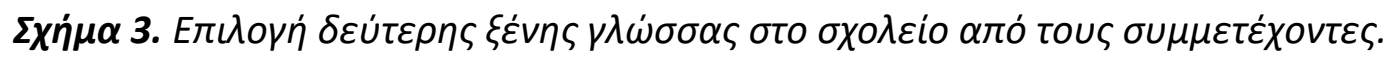

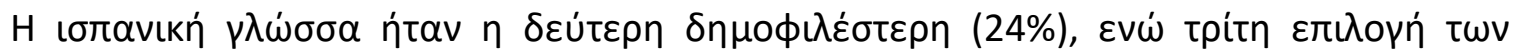

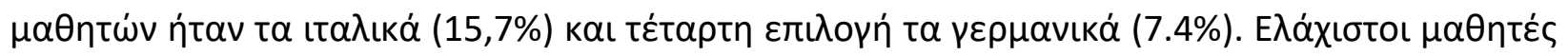

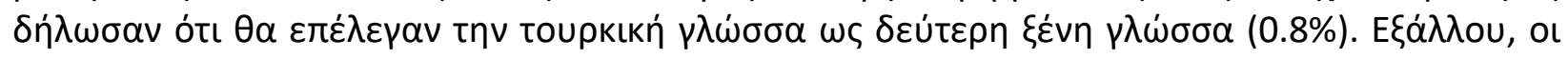

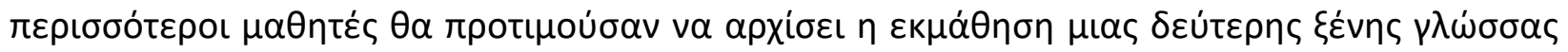

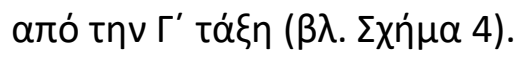

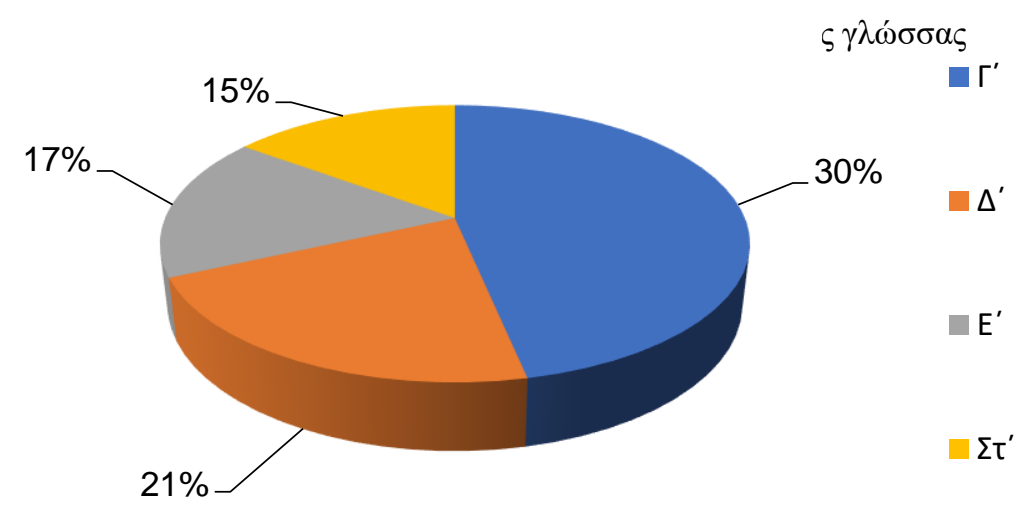

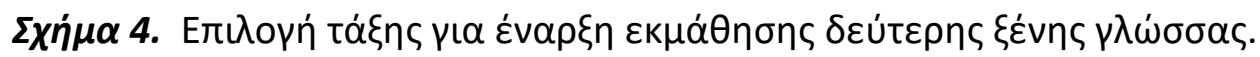


MULTILINGUAL ACADEMIC JOURNAL OF EDUCATION AND SOCIAL SCIENCES

Vol. 1 No. 1, 2013, E-ISSN: 2308-0876 @ 2013 KWP

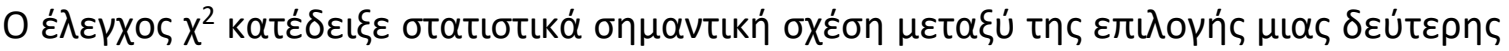

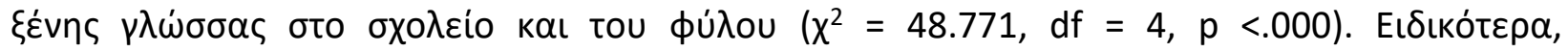

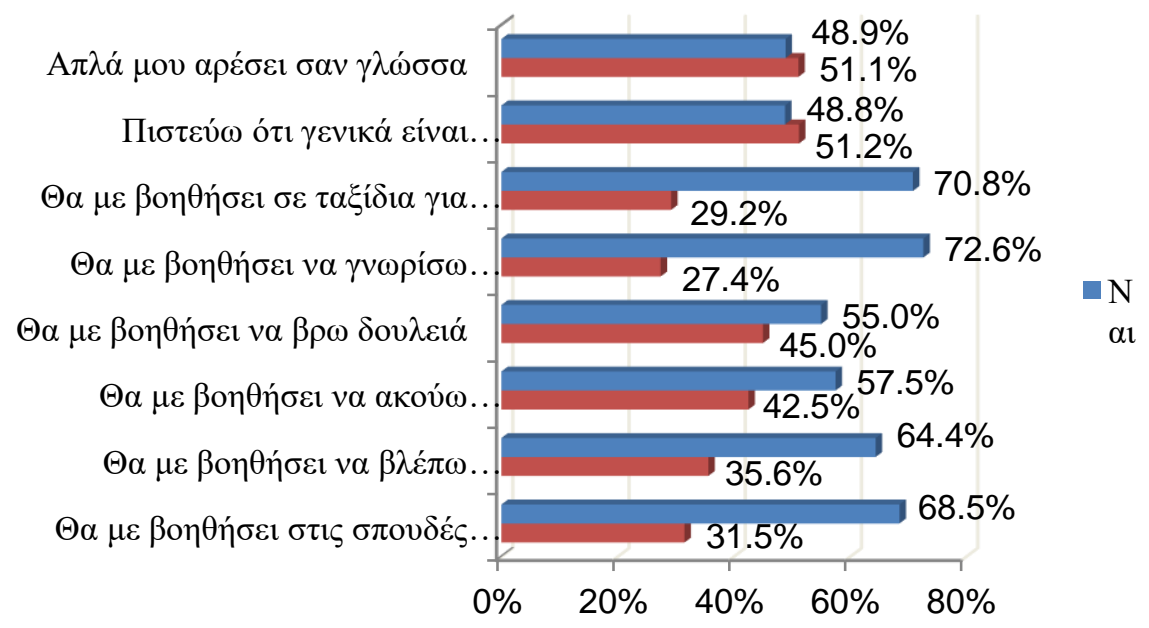

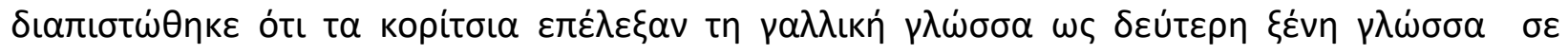

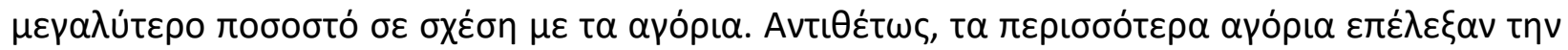

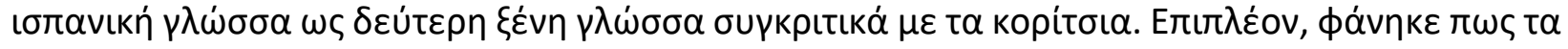

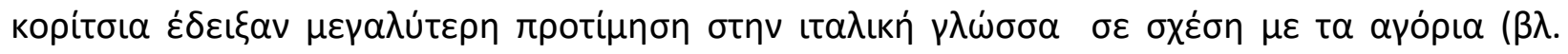

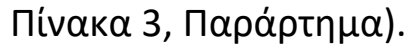

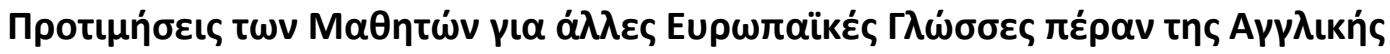

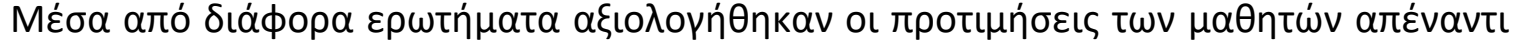

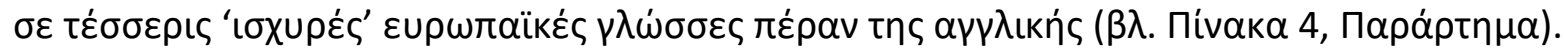

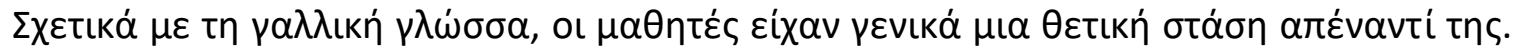

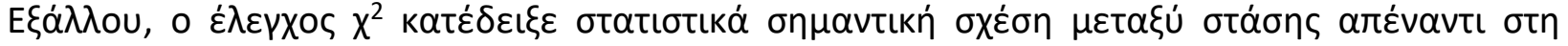

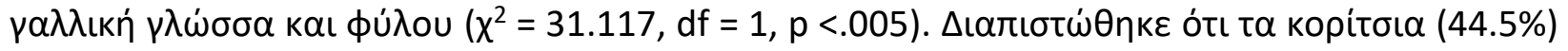

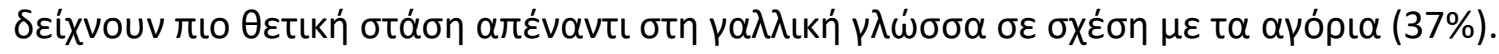

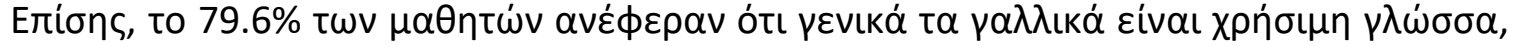

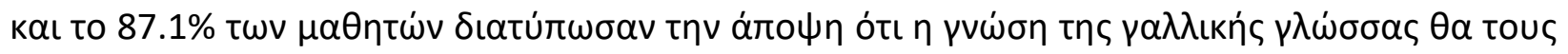

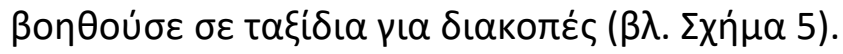


MULTILINGUAL ACADEMIC JOURNAL OF EDUCATION AND SOCIAL SCIENCES

Vol. 1 No. 1, 2013, E-ISSN: 2308-0876 @ 2013 KWP

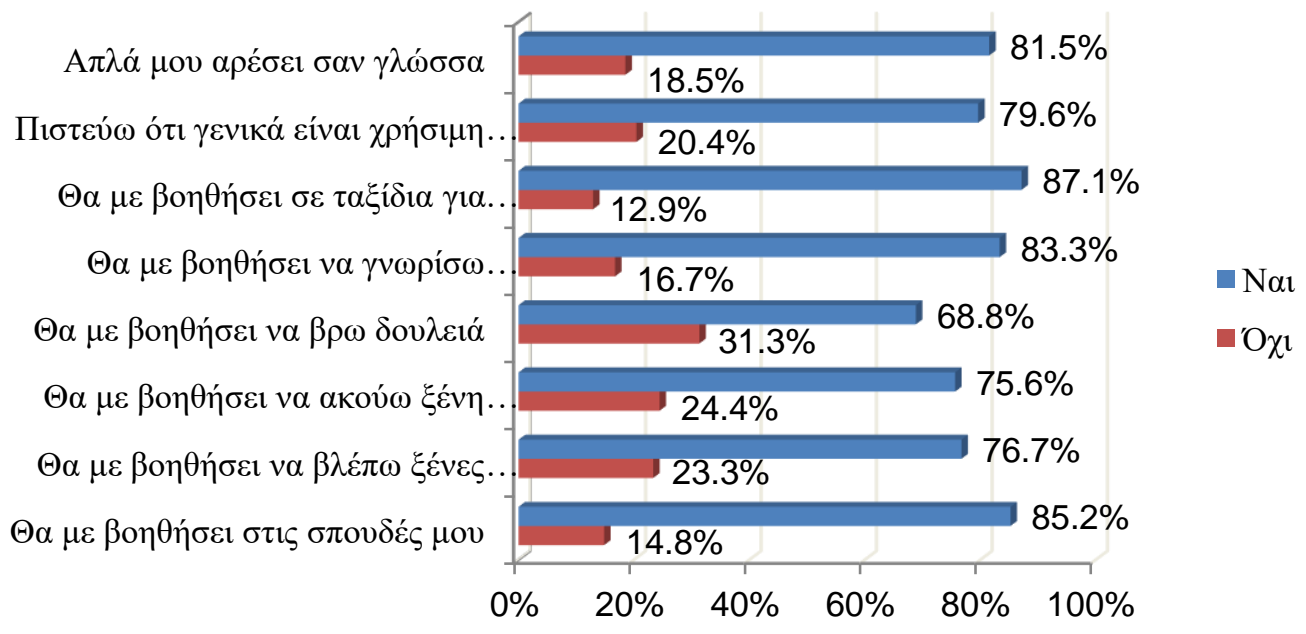

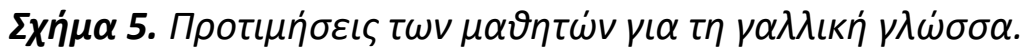

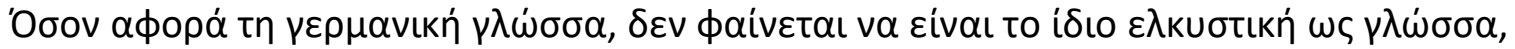

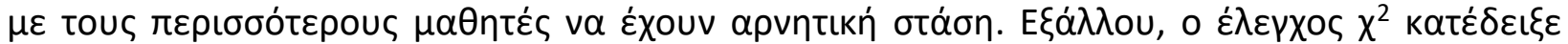

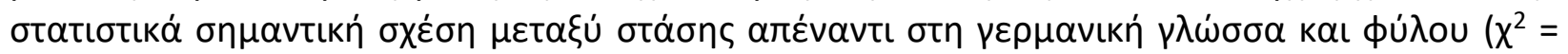

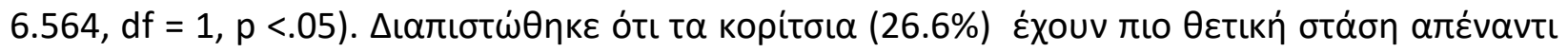

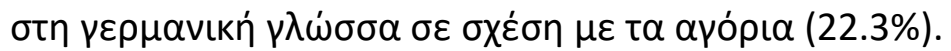

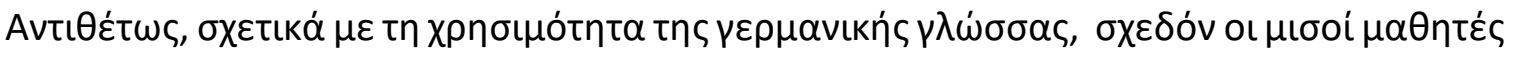

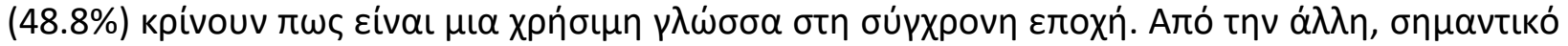

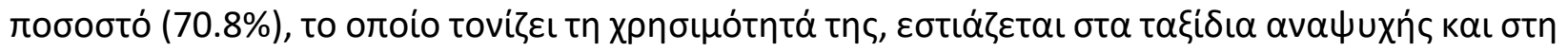

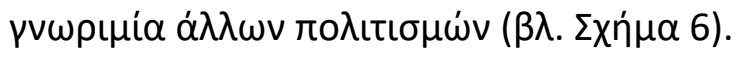

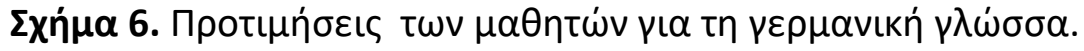

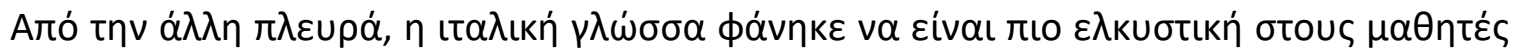

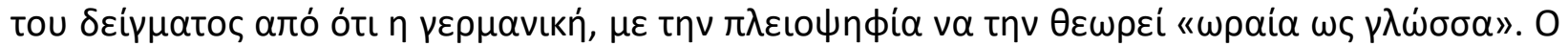

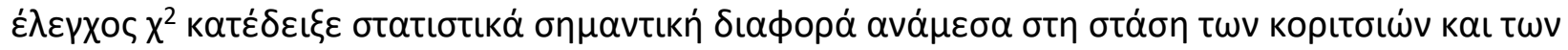

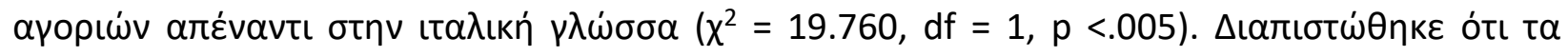

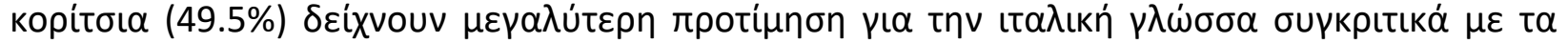
aүópıа (22.9\%).

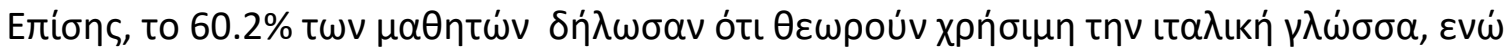
$\eta \pi \lambda \varepsilon เ$

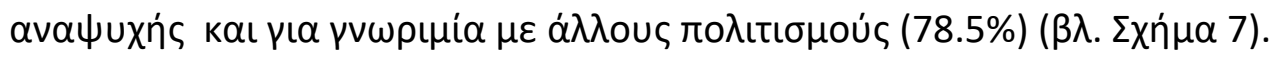


MULTILINGUAL ACADEMIC JOURNAL OF EDUCATION AND SOCIAL SCIENCES

Vol. 1 No. 1, 2013, E-ISSN: 2308-0876 @ 2013 KWP

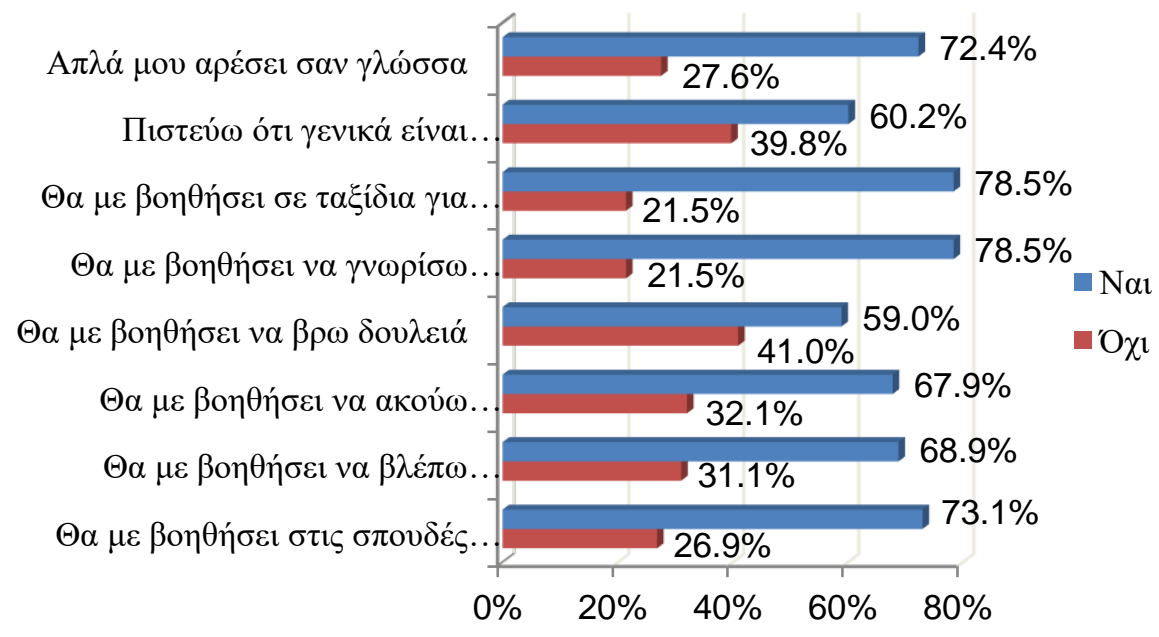

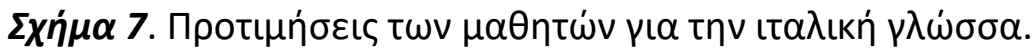

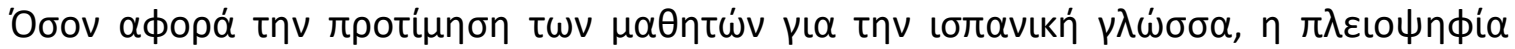

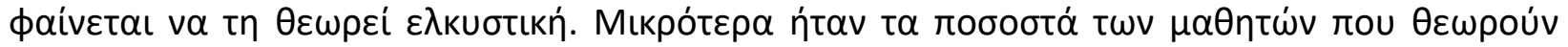

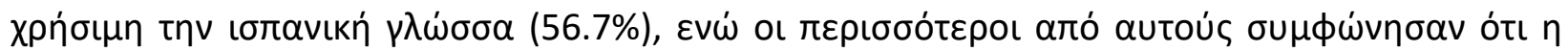

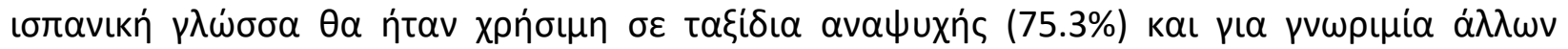

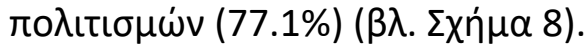
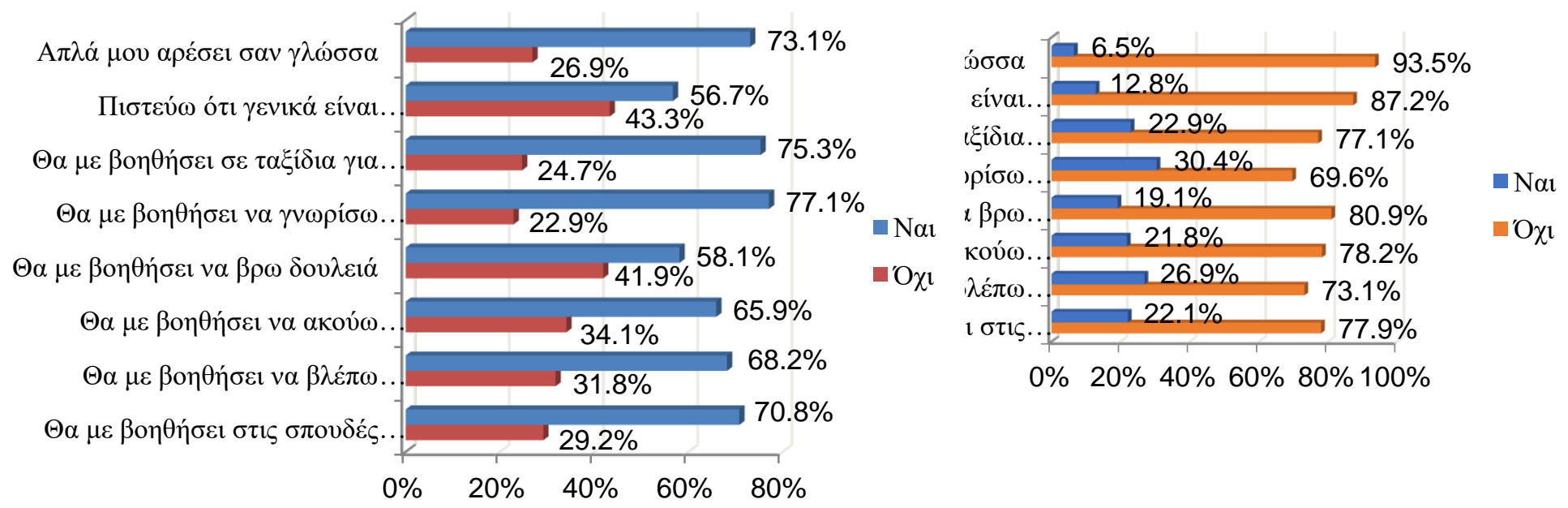

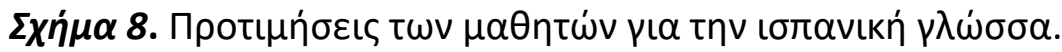

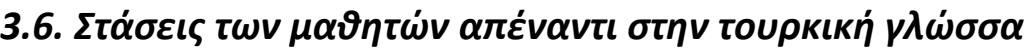

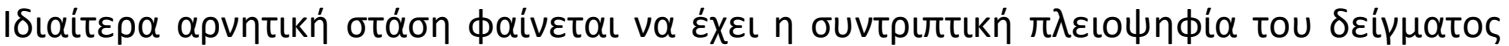

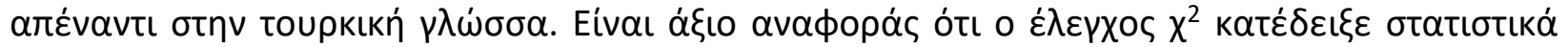

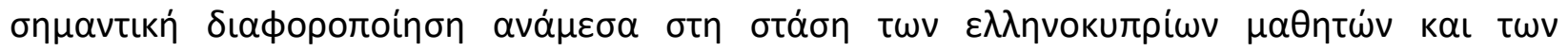

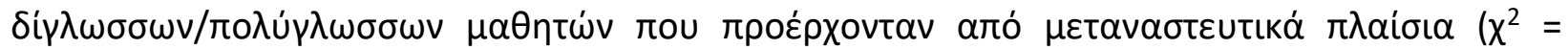

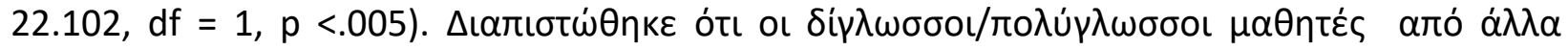


MULTILINGUAL ACADEMIC JOURNAL OF EDUCATION AND SOCIAL SCIENCES

Vol. 1 No. 1, 2013, E-ISSN: 2308-0876 @ 2013 KWP

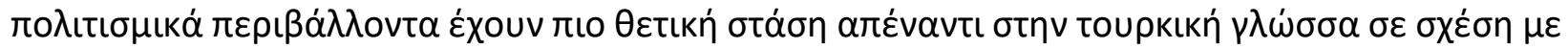

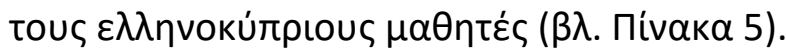

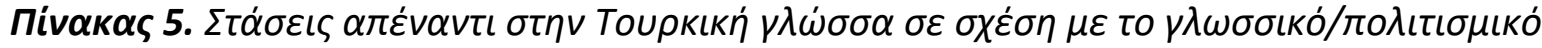
$\pi \rho \circ \varphi i \lambda$

\begin{tabular}{|c|c|c|c|c|c|}
\hline \multirow{2}{*}{ 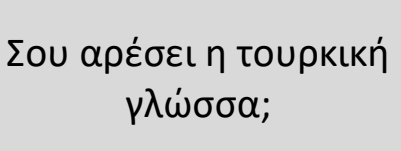 } & \multicolumn{2}{|c|}{$\mathrm{Nal}$} & \multicolumn{2}{|c|}{ 'Oxı } & \multirow[b]{2}{*}{ 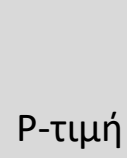 } \\
\hline & $\mathrm{N}$ & $\%$ & $\mathrm{~N}$ & $\%$ & \\
\hline \multicolumn{6}{|l|}{ 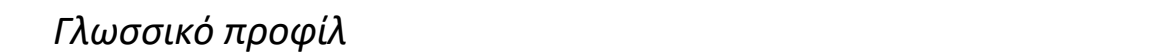 } \\
\hline 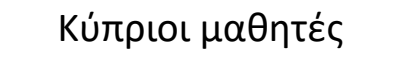 & 44 & 3.7 & 884 & 74.7 & \\
\hline 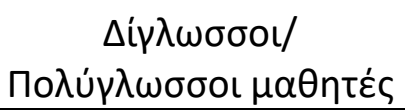 & 33 & 2.8 & 222 & 18.8 & $<0.005$ \\
\hline
\end{tabular}

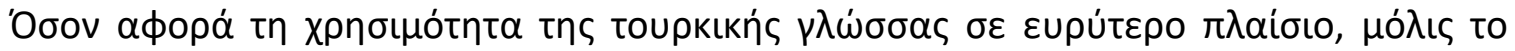

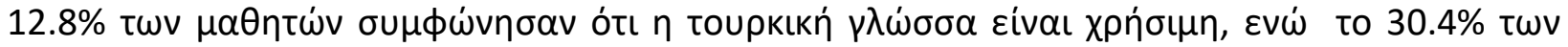

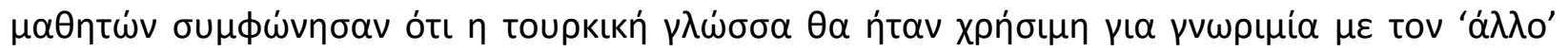

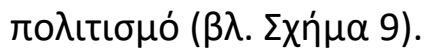

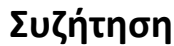

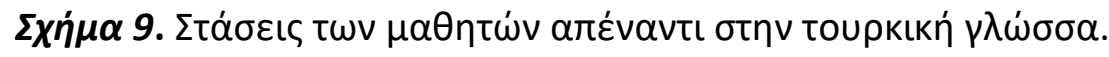

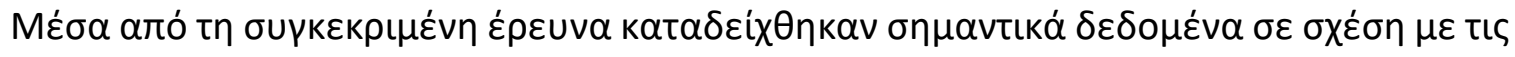

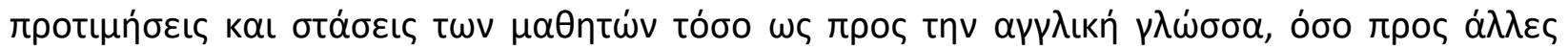

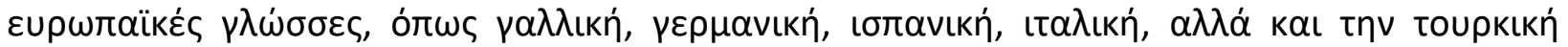

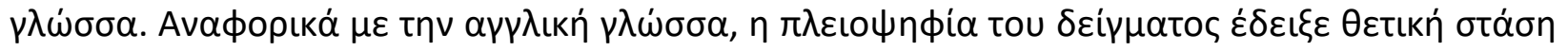

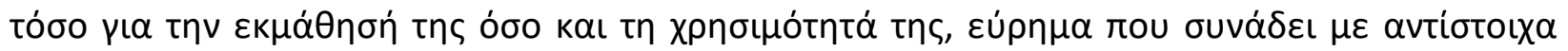

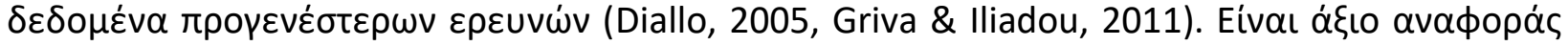

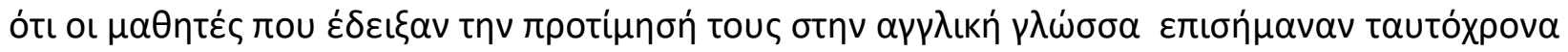

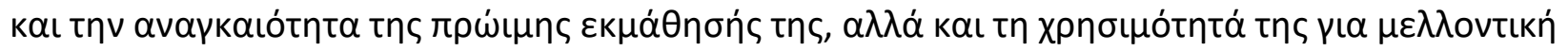

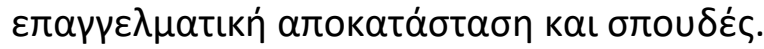

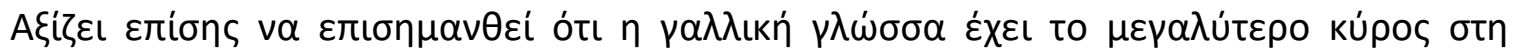

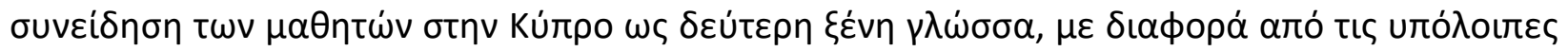

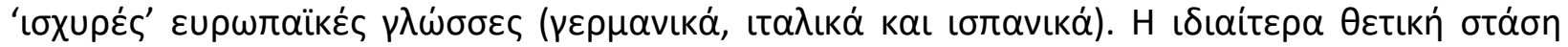

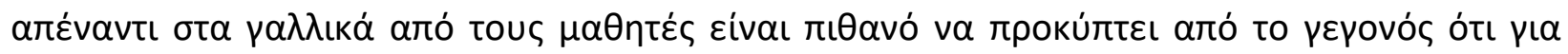

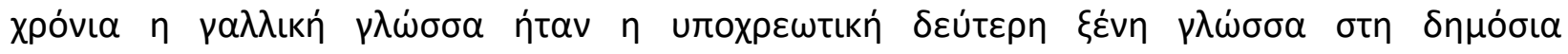

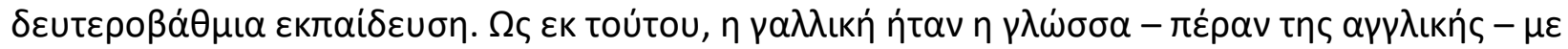

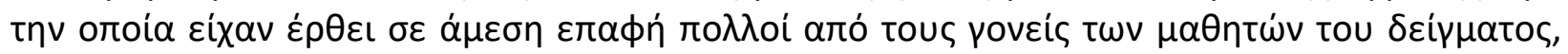

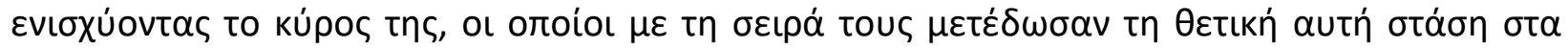
$\pi \alpha \iota \delta ı \alpha$ touৎ.

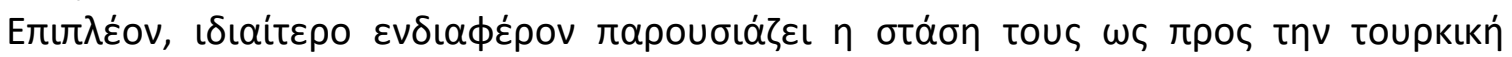

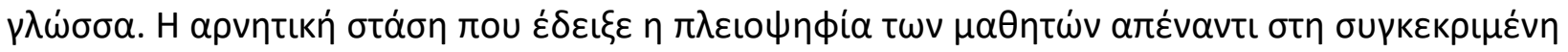

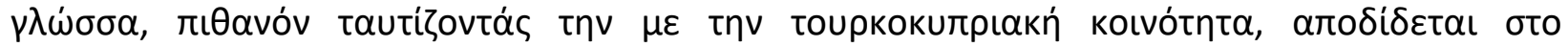

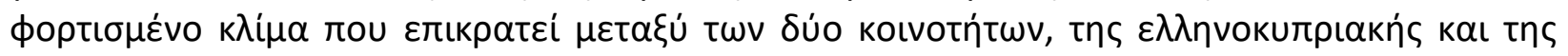


MULTILINGUAL ACADEMIC JOURNAL OF EDUCATION AND SOCIAL SCIENCES

Vol. 1 No. 1, 2013, E-ISSN: 2308-0876 @ 2013 KWP

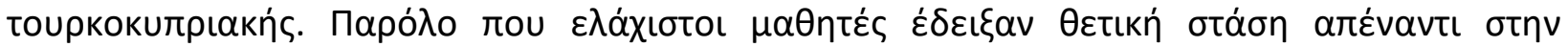

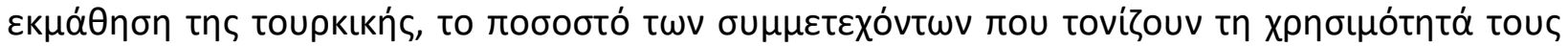

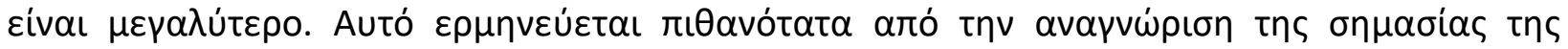

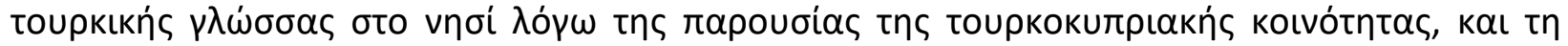

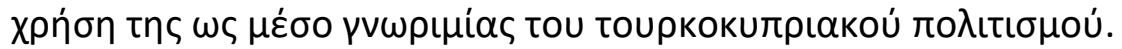

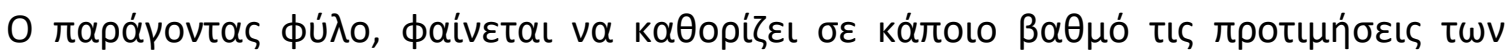

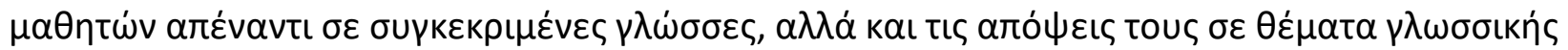

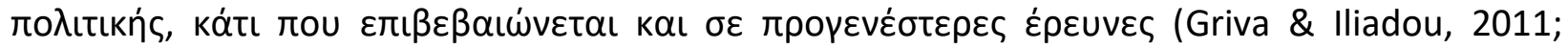
Gardner, 1985; Larocque, 2006).

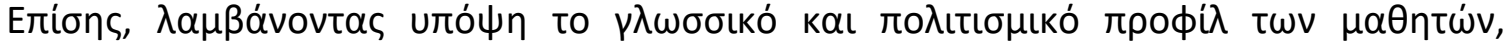

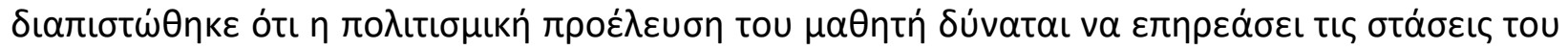

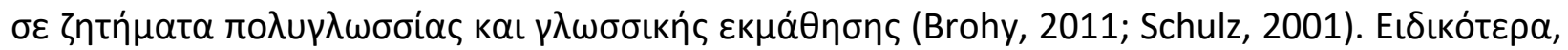

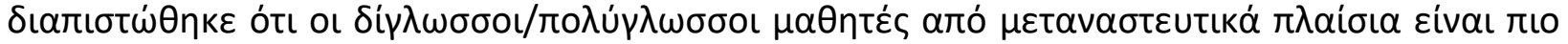

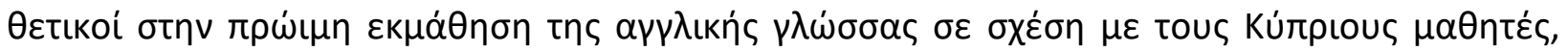

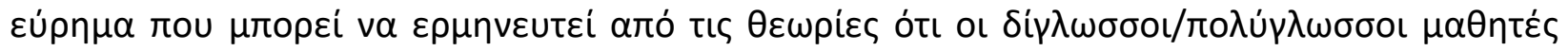

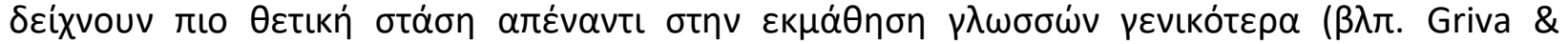
Chostelidou, 2011; Nikolov \& Curtain, 2000; Pinter, 2006.).

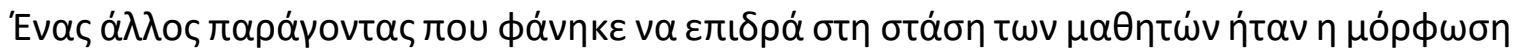

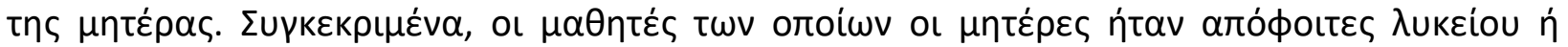

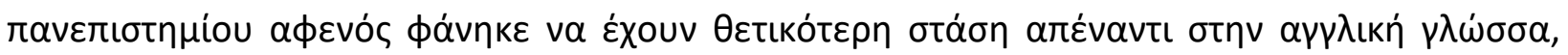

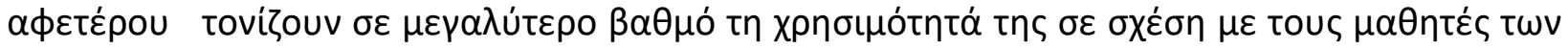

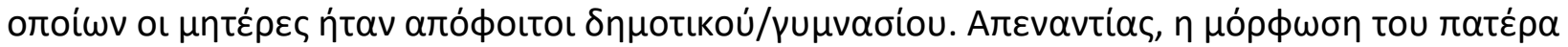

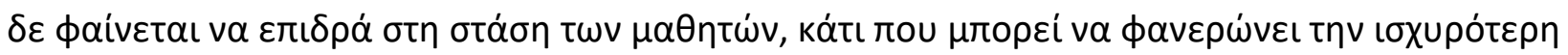

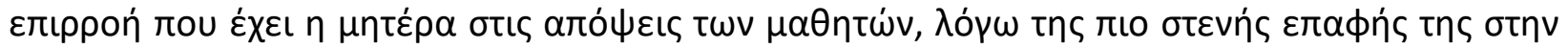

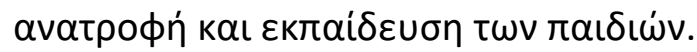

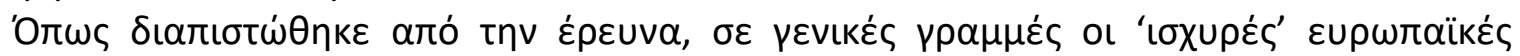

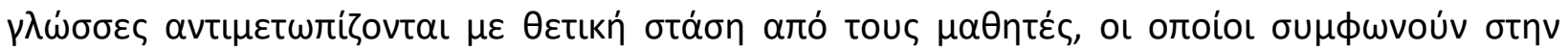

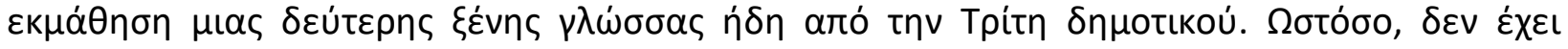

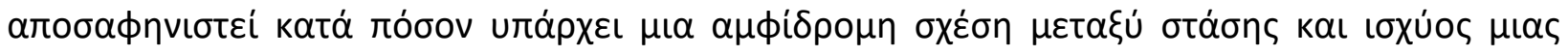

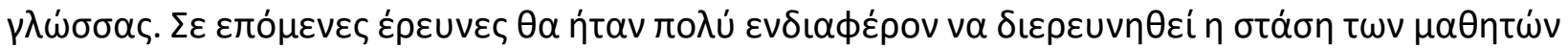

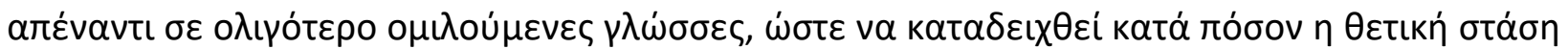

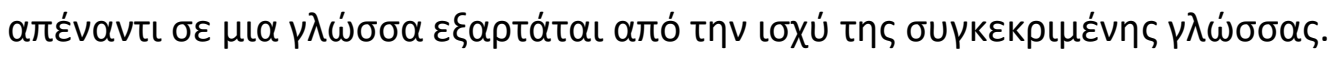

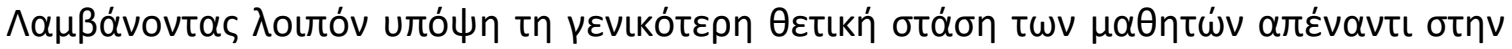

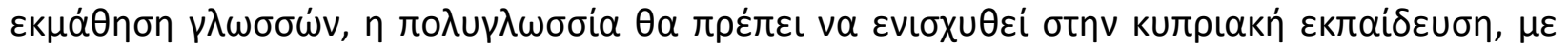

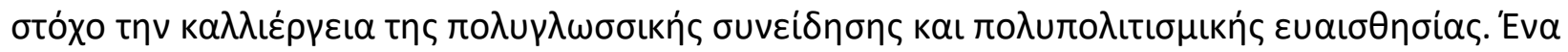

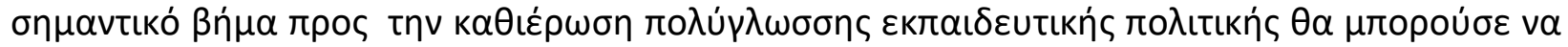

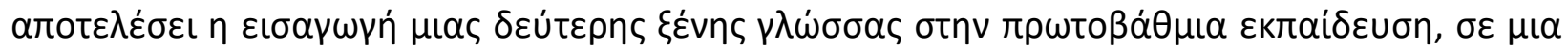

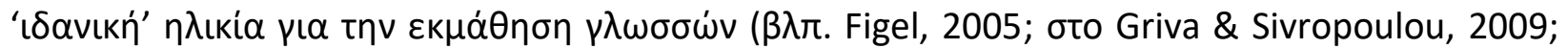
Lightbown \& Spada, 2006; Singleton, 2000).

\section{References}

Bacon, S. M., \& Finnemann, M. (1992). Sex differences in self-reported beliefs about foreign language learning and authentic oral and written input. Language Learning, 42, 471-495. 
MULTILINGUAL ACADEMIC JOURNAL OF EDUCATION AND SOCIAL SCIENCES

Vol. 1 No. 1, 2013, E-ISSN: 2308-0876 @ 2013 KWP

Bernat, E., \& Lloyd, R. (2007). Exploring the gender effect on EFL learners' beliefs about language learning. Australian Journal of Educational \& Developmental Psychology, 7, 79-91.

Breen, M. P. (2001). Learner contributions to language learning: New directions in research. Essex: Pearson Education Limited.

Brohy, C. (2001). Generic and/or specific advantages of bilingualism in a dynamic plurilingual situation: The case of French as official L3 in the school of Samedan (Switzerland). International Journal of Bilingual Education and Bilingualism, 4(1), 38-49.

CEF. (2001). Common European Framework of Reference for Languages: Learning, teaching, assessment, Cambridge: Cambridge University Press.

Csizer, K., \& Dornyei, Z. (2005). The internal structure of language learning motivation and its relationship with language choice and learning effort. The Modern Language Journal, 89 (1), 19-36.

Diallo, I. (2005). Language planning, language-in-education policy and attitudes towards

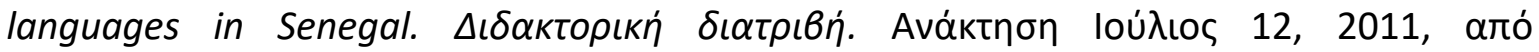
http://www4.gu.edu.au:8080/adt-root/uploads/approved/adt-

QGU20070105.113405/public/01Front.pdf

Eacea. (2009). Study on the impact of information and communications technology (ICT) and New

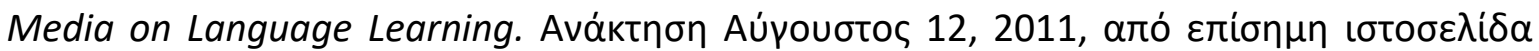

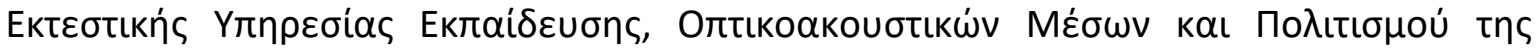

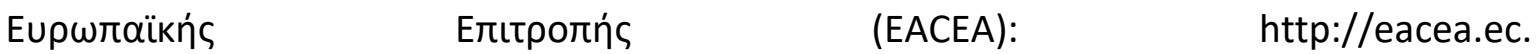
europa.eu/Ilp/studies/documents/study_impact_ict_new_media_language_learning/final _report_en.pdf

Edelenbos, P., Johnstone, J., \& Kubanek, A. (2006). The main pedagogical principles underlying the teaching of languages to very young learners: Languages for the children of Europe. European Commission.

European Commission. (2004). Many tongues, one family. Languages in the European Union. Directorate General Press and Communication.

Gardner, R. C. (1985). Social Psychology and Second Language Learning: The role of attitude and motivation. London: Edward Arnold.

Griva, E., \& Sivropoulou E. (2009). Implementation and evaluation of an early foreign language project in kindergarten, The Early Childhood Journal, 37(1), 79-87.

Griva, E., \& Chostelidou, D. (2011). Multilingual Learning: A Comparative Study of Students Learning FLs in Greek Primary and Secondary Education. ¿to J. S. Avery, \& M. H. Stewart

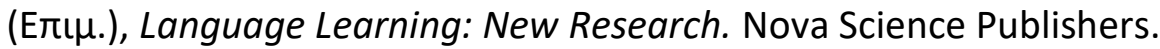

Griva, E., \& Iliadou, S. (2011). Teachers' viewpoints and students attitudes towards plurilingualism. European Journal of Language Policy, 15-36.

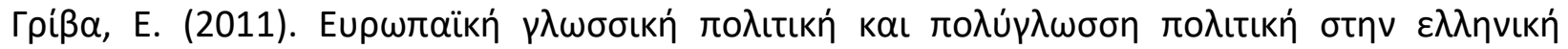

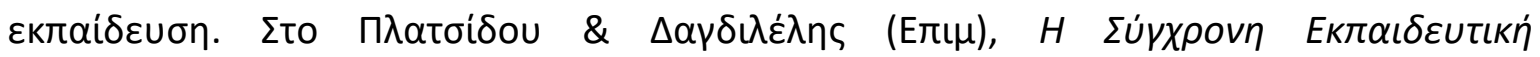

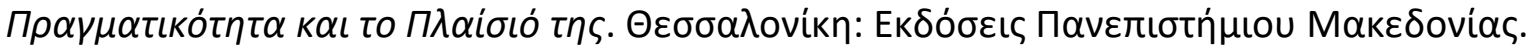

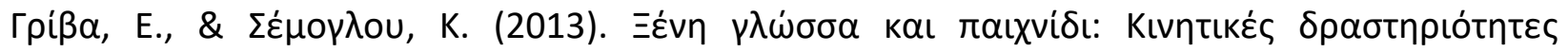

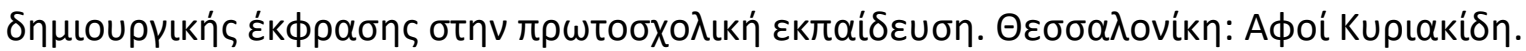

Horwitz, E. (1988). The beliefs about language learning of beginning university foreign language students. Journal of Modern Language , 72 (3), 283-294. 
MULTILINGUAL ACADEMIC JOURNAL OF EDUCATION AND SOCIAL SCIENCES

Vol. 1 No. 1, 2013, E-ISSN: 2308-0876 @ 2013 KWP

Kara, A. (2009). The Effect of a 'Learning Theories' Unit on Students' Attitudes towards Learning. AustralianJournal of Teacher Education, 34(3), 100-113.

Kern, R. G. (1995). Students' and teachers' beliefs about language learning. Foreign Language Annals, 28, 71-92.

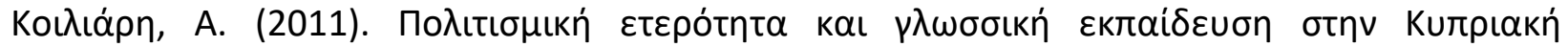

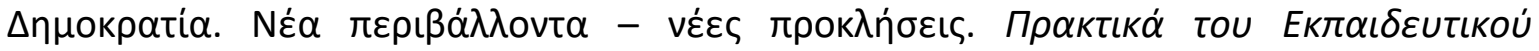

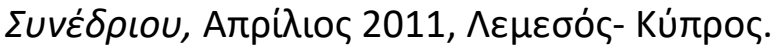

Kuhlemeir, H., Bergh, H., \& Melse, L. (1996). Attitudes and achievements in the first year German language instruction in Dutch secondary education. The Modern Language Journal, 80, 494508.

Larocque, N. (2006). Les attitudes des élèves envers l'apprentissage du français dans une école,

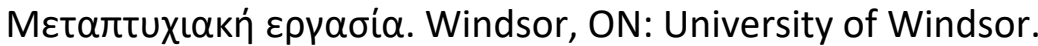

Lightbown, P. M., \& Spada, N. (2006). How Languages are Learned. New York: Oxford University Press.

Nikolov, M., \& Curtain H. (2000). An Early Start: Young Learners and Modern Languages in Europe and Beyond. Council of Europe: European Centre for Modern Languages.

Pinter, A. (2006). Teaching Young Language Learners. Oxford University Press.

Sakui, K., \& Gaies, S. J. (1999). Investigating Japanese learners' beliefs about language learning. System, 27, 473-492.

Schulz, R. A. (2001). Cultural differences in students and teacher perceptions concerning the role of grammar instruction and corrective feedback: USA-Columbia. The Modern Language Journal, 85, 244-285.

Siebert, L. L. (2003). Student and teacher beliefs about language learning. The ORTESOL Journal, 21, 7-39.

Singleton, D. (2001). Age and second language acquisition. Annual Review of Applied Linguistics, 21, 77-91.

Victori, M., \& Lockhart, W. (1995). Enhancing metacognition in self-directed language learning. System, 23, 223-234.

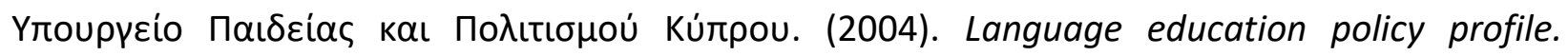

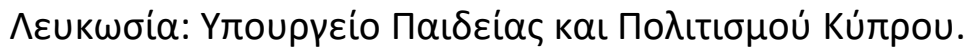

Dr. Eleni Griva is an Assistant Professor at the Faculty of Education - University of Western Macedonia. Her research interests include: L2/FL Learning and Teaching, Language learning strategies, Bilingualism/multilingualism, Language policy. She has published three books, and she has been author or coauthor of over 100 papers in journals, collected editions and conference proceedings and she has participated in various projects and international conferences.

Panayotis Panteli, MA in Education Studies. He is a primary school teacher and has participated in various projects and conferences. He has been coauthor of papers in journals and conference proceedings

Eleni Tsakiridou is Associate Professor of Applied Statistics and Educational Research at the Department of Primary Education of the University of Western Macedonia in Greece. She is currently vice chairperson in the Department of Informatics and Telecommunications 


\section{MULTILINGUAL ACADEMIC JOURNAL OF EDUCATION AND SOCIAL SCIENCES \\ Vol. 1 No. 1, 2013, E-ISSN: 2308-0876 2013 KWP}

Engineering of the University of Western Macedonia. She has published in more than 80 Greek and international refereed journals, in collective volumes and proceedings. Her research interests include Applied Statistics, Educational Research, Economics of Education, Efficiency and Equity Matters and Operations Research. She is also interested in population structure studies and development issues, sampling, data collection and data analysis.П $\alpha \rho \alpha \rho \tau \eta \mu \alpha$

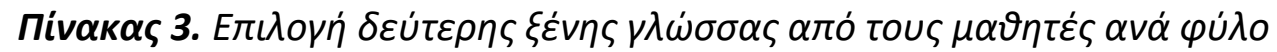

\begin{tabular}{|c|c|c|c|c|c|c|c|c|c|c|c|}
\hline$\Gamma \lambda \omega \dot{\sigma} \sigma \alpha$ & \multicolumn{2}{|c|}{$\Gamma \alpha \lambda \lambda \iota \kappa \dot{\alpha}$} & \multicolumn{2}{|c|}{ 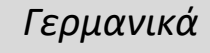 } & \multicolumn{2}{|c|}{ Точркцка́ } & \multicolumn{2}{|c|}{ 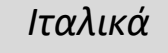 } & \multicolumn{2}{|c|}{ 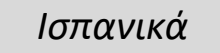 } & \multirow[b]{2}{*}{$\mathrm{P}-\tau \iota \mu \dot{n}^{\prime}$} \\
\hline 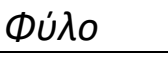 & $\mathrm{N}$ & $\%$ & $\mathrm{~N}$ & $\%$ & $\mathrm{~N}$ & $\%$ & $\mathrm{~N}$ & $\%$ & $\mathrm{~N}$ & $\%$ & \\
\hline Aүópıа & 271 & 21.8 & 51 & 4.1 & 8 & 0.6 & 84 & 6.8 & 196 & 15.8 & \\
\hline Корі́тбı & 365 & 29.4 & 43 & 3.5 & 2 & 0.2 & $\begin{array}{l}11 \\
5\end{array}$ & 9.3 & 107 & 8.6 & $<0.005$ \\
\hline
\end{tabular}

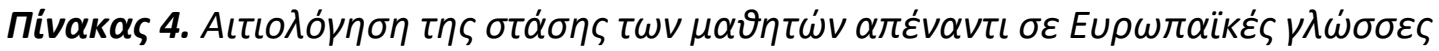

\begin{tabular}{|c|c|c|c|c|c|c|c|c|}
\hline & \multicolumn{2}{|c|}{ 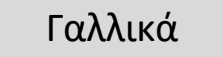 } & \multicolumn{2}{|c|}{ 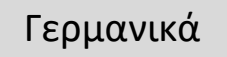 } & \multicolumn{2}{|c|}{ 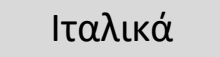 } & \multicolumn{2}{|c|}{ 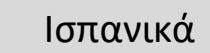 } \\
\hline & $\begin{array}{l}\mathrm{Nal} \\
(\%)\end{array}$ & $\begin{array}{l}\text { 'OXı } \\
(\%)\end{array}$ & $\begin{array}{l}\mathrm{Nal} \\
(\%)\end{array}$ & $\begin{array}{l}\text { 'OXı } \\
(\%)\end{array}$ & $\begin{array}{l}\mathrm{Nal} \\
(\%)\end{array}$ & $\begin{array}{l}\mathrm{O} \chi \mathrm{L} \\
(\%)\end{array}$ & $\begin{array}{l}\mathrm{Nal} \\
(\%)\end{array}$ & $\begin{array}{l}\text { 'OXı } \\
(\%)\end{array}$ \\
\hline 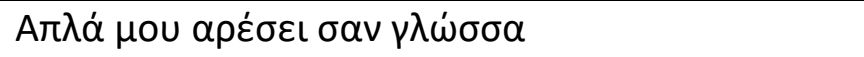 & 81.5 & 18.5 & 48.9 & 51.1 & 72.4 & 27.6 & 73.1 & 26.9 \\
\hline 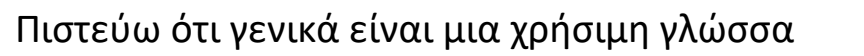 & 79.6 & 20.4 & 48.8 & 51.2 & 60.2 & 39.8 & 56.7 & 43.3 \\
\hline 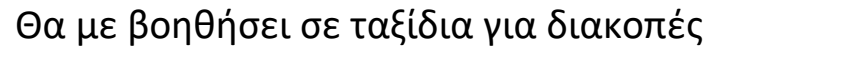 & 87.1 & 12.9 & 70.8 & 29.2 & 78.5 & 21.5 & 75.3 & 24.7 \\
\hline 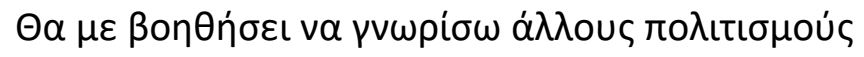 & 83.3 & 16.7 & 72.6 & 27.4 & 78.5 & 21.5 & 77.1 & 22.9 \\
\hline 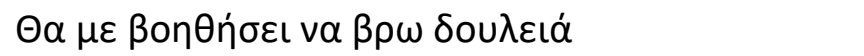 & 68.8 & 31.3 & 55.0 & 45.0 & 59.0 & 41.0 & 58.1 & 41.9 \\
\hline 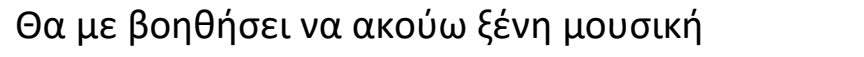 & 75.6 & 24.4 & 57.5 & 42.5 & 67.9 & 32.1 & 65.9 & 34.1 \\
\hline 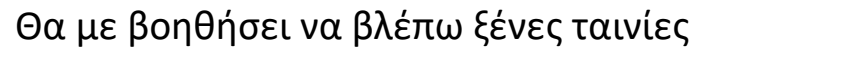 & 76.7 & 23.3 & 64.4 & 35.6 & 68.9 & 31.1 & 68.2 & 31.8 \\
\hline 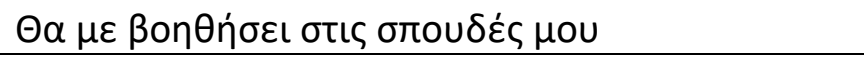 & 85.2 & 14.8 & 68.5 & 31.5 & 73.1 & 26.9 & 70.8 & 29.2 \\
\hline
\end{tabular}

